\title{
CHOICE OF MEASURE SOURCE TERMS IN INTERFACE COUPLING FOR A MODEL PROBLEM IN GAS DYNAMICS
}

\author{
FRÉDÉRIC COQUEL, EDWIGE GODLEWSKI, KHALIL HADDAOUI, \\ CLAUDE MARMIGNON, AND FLORENT RENAC
}

\begin{abstract}
This paper is devoted to the mathematical and numerical analysis of a coupling procedure for one-dimensional Euler systems. The two systems have different closure laws and are coupled through a thin fixed interface. Following the work of Ambroso, Chalons, Coquel and Galié (2004), we propose to couple these systems by a bounded vector-valued Dirac measure, concentrated at the coupling interface, which in the applications may have a physical meaning. We show that the proposed framework allows the control of the coupling conditions and we propose an approximate Riemann solver based on a relaxation approach preserving equilibrium solutions of the coupled problem. Numerical experiments in constrained optimization problems are then presented to assess the performances of the present method.
\end{abstract}

\section{INTRODUCTION}

The study of large-scale and complex problems exhibiting a wide range of physical space and time scales (see for instance [14, 35, 62 ), usually requires separate solvers adapted to the resolution of specific scales. This is the case of many industrial flows. Let us quote, for example, the numerical simulation of two-phase flows applied to the burning liquid oxygen-hydrogen gas in rocket engines [58]. This kind of flow contains both separated and dispersed two-phase flows, due to atomization and evaporation phenomena. This requires appropriate models and solvers for separated and dispersed phases that have to be appropriately coupled. Another example concerns turbomachine flows which can be modeled by the Euler equations of gas dynamics with different closure laws between the stages of the turbine, where the conditions of temperature and pressure are strongly heterogeneous. The coupling of these different systems is thus necessary to give a complete description of the flow inside the whole turbine.

The method of interface coupling allows us to represent the evolution of such flows, where different models are separated by fixed interfaces. First, coupling conditions are specified at the interface to exchange information between the systems. The definition of transmission conditions generally results from physical consideration, e.g., the conservation or the continuity of given variables. Then, the transmission conditions are represented at the discrete level. The study of interface coupling for nonlinear hyperbolic systems has received attention for several years. In [43], the authors study the scalar case from both mathematical and numerical

Received by the editor April 25, 2014 and, in revised form, November 21, 2014 and January 23, 2015.

2010 Mathematics Subject Classification. Primary 35L04, 76M12, 76N15.

Key words and phrases. Model coupling, hyperbolic systems of conservation laws, measure source term, Riemann solver, relaxation, constrained optimization. 
points of view. They couple two different one-dimensional conservation laws at a fixed interface. The case of systems of conservation laws has been considered in [4], where admissible coupling conditions are defined at the interface. Indeed, since the systems we consider are hyperbolic, the boundary conditions must be imposed in a weak sense 29 .

From previous studies, coupling conditions [4,6, 8, 10, 18, 23, 34, 39, 40, 43, 50, 52 , 56. can be classified in three categories: flux coupling, state coupling and coupling with measure source term. The flux coupling method is a conservative approach which ensures the continuity of the physical flux through the coupling interface. Conversely, the state coupling method is a nonconservative approach which imposes (at least weakly) the continuity property of either the (conservative) variables or a nonlinear transformation of them (say, primitive variables). Finally, the coupling condition can be modelled thanks to a bounded vector-valued Dirac measure concentrated at the coupling interface [4, 5, 34, 40. The coupling condition is then prescribed from the definition of the mass of the measure. This modelling presents several advantages. It allows the consideration of both conservative and state couplings. Moreover, it enables the control of the coupling conditions by taking into account the mass, momentum or energy losses located through the discontinuity.

In this study, we focus on a model case which concerns the coupling of gas dynamics equations with different closure laws for the pressure. We propose an extension of the works [4,5,34, to the full Euler system (with energy) in the general case where all the source terms may be nonzero. Indeed, in these previous contributions, the authors assumed the strict conservation of the density. The motivation of this present study is to extend the panel of applications, and the computations we present do involve test cases involving the energy equation which could not be covered in the previous work. These test cases are motivated by the applications among which the modeling of some sophisticated control devices are meant to protect instruments from damages due to overheating. A few references on discharge relationships and component head losses for hydraulic structures or the modeling of pressure drop are already given in [5] (see references [19, 34, 36, 37] therein). Some devices for the flow control involve the momentum and impact the energy conservation. Note, however, that the topic of flow control is much broader and the simple models we are considering are but a small step in this direction for improving the simulation (see [32,33] concerning aerospace for instance). One may also mention in the context of oil recovery the possibility of drawing out and reinjecting elsewhere a certain amount of gas (which is referred to as gas-lift and active bypass, see [30]), in order to prevent severe slugging, and this may be modeled as point losses in the mass conservation equation.

Indeed, an important remark is that the above mentioned devices are localized and small with respect to some characteristic length of the flow. Hence, in the modeling of the situation by a system of PDE, a natural scaling of the equations yields a point source at the interface, which means a multiple of a Dirac measure, classically placed at the origin. Following [36, 37, this can be interpreted as a scattering problem, with nonlinear waves interacting on a large scale compared to the scale of the interface which is then considered as infinitely thin.

Then, as explained in [37, the solution of the Riemann problem for the, let us say, simplified scaled model gives to the leading order the large time behavior of the full system with unscaled source. Thus, the study of Riemann problems 
comes naturally from the study of the interaction of nonlinear localized waves, and following this scattering setting, the Riemann solution represents the outgoing wave operator.

We show that the present framework is fairly flexible. In particular, it allows us to handle coupling problems constrained by the property that the Riemann solutions should keep their values in a given set while minimizing some convex nonlinear cost functions built on the source terms at the interface. These cost functions are defined by physical motivations.

Before we conclude this introduction, let us mention some very interesting works which, by some aspects, are strongly related to the present one. Interface coupling is clearly related to the study of conservation laws with discontinuous fluxes. In the scalar case, it is possible to give a rigorous framework in which existence and uniqueness results hold [11. Then, singular source terms appear in different contexts which may correspond either to some (more or less) physical modelling as in [28] or [12,13] or to numerical devices. Indeed, measure source terms are naturally introduced in many approaches for the construction of well-balanced schemes for hyperbolic systems with geometric source terms [45] and, in turn, there is a natural link with the analysis of resonant systems 38] (and references therein). If general theoretical existence and uniqueness results may be obtained in the scalar case, for instance, $L^{1}$ stability results in the nonresonant case [49] or partial results in the resonant case [2], also for the existence of traces [54,61], it is beyond the scope of the present work to give a rigorous notion of solution and well-posedness for the general coupling Cauchy problem in the case of systems. We rather focus on the solution of Riemann problems with coupling, which as mentioned above do represent at some scale the result of nonlinear wave interactions and, moreover, are naturally involved in the numerical approach, and on the optimization of source terms. Last, even if the link is not yet as close with interfacial coupling as the above subjects are, we should however mention the control of nonlinear hyperbolic systems [19] a subject appearing naturally with flow in networks [3].

The paper is organized as follows. The coupling problem is introduced in 92 , The numerical approach to solve this coupling problem is described in $₫ 3$. The method we consider relies on a relaxation approximation which is presented before the associated Riemann problem is solved. Then the source terms are chosen in order to preserve steady solutions of the coupled problem. Strategies for the dynamic evaluation of the source terms satisfying various criteria are then given. Several numerical experiments based on Riemann problems associated to the coupling model are then described in 8 . Finally, the last section summarizes the conclusions of this study.

\section{Coupling With measure source term}

2.1. Physical problem and governing equations. We focus on the one-dimensional problem of interface coupling between two hyperbolic systems of conservation laws. More precisely, we consider the systems of Euler equations that model the flow of a compressible polytropic ideal gas. Both models are separated in space by a thin interface localized at $x=0$ and differ from their definition of the pressure. The flow is described by a system $\mathcal{S}_{L}$ in the left domain $\mathcal{D}_{L}=\{x<0, t>0\}$ and by a system $\mathcal{S}_{R}$ in the right domain $\mathcal{D}_{R}=\{x>0, t>0\}$. We look for a function 
$\mathbf{u}:(x, t) \in \mathbb{R} \times \mathbb{R}^{+} \mapsto \mathbf{u}(x, t) \in \Omega \subset \mathbb{R}^{3}$, the solution of

$$
\begin{array}{lll}
\mathcal{S}_{L}: & \partial_{t} \mathbf{u}+\partial_{x} \mathbf{f}_{L}(\mathbf{u})=0, & (x, t) \in \mathcal{D}_{L}, \\
\mathcal{S}_{R}: & \partial_{t} \mathbf{u}+\partial_{x} \mathbf{f}_{R}(\mathbf{u})=0, & (x, t) \in \mathcal{D}_{R},
\end{array}
$$

with initial condition

$$
\mathbf{u}(x, 0)=\mathbf{u}_{0}(x), \quad x \in \mathbb{R} .
$$

The conservative variable vector and smooth flux functions are defined by

$$
\mathbf{u}=\left(\begin{array}{c}
\rho \\
\rho u \\
\rho E
\end{array}\right) \quad \text { and } \quad \mathbf{f}_{\alpha}(\mathbf{u})=\left(\begin{array}{c}
\rho u \\
\rho u^{2}+p_{\alpha}(\tau, \varepsilon) \\
\rho E u+p_{\alpha}(\tau, \varepsilon) u
\end{array}\right), \quad \alpha=L, R .
$$

The variables $\rho, \tau=1 / \rho$ and $\varepsilon=\varepsilon(\tau, s)$ stand for the density, the specific volume and the specific internal energy of the gas, respectively, where $s$ denotes the physical entropy. The variable $u$ denotes the velocity and $E=\varepsilon+u^{2} / 2$ the specific total energy.

The thermodynamic variables are related by two ideal gas equations of state

$$
p_{\alpha}(\tau, \varepsilon)=\frac{\left(\gamma_{\alpha}-1\right) \varepsilon}{\tau}, \quad \alpha=L, R,
$$

where $\gamma_{L}$ and $\gamma_{R}$ denote the respective ratio of specific heats (satisfying $\gamma_{\alpha}>1$ ). Introducing the sound speed in the fluid

$$
c_{\alpha}=c_{\alpha}(\tau, s)=\sqrt{\frac{\gamma_{\alpha} p_{\alpha}}{\rho}}, \quad \alpha=L, R,
$$

the pressure laws are assumed to verify the inequalities

$$
p_{\alpha}>0, \quad\left(\frac{\partial p_{\alpha}}{\partial \tau}\right)_{s}=-\rho^{2} c_{\alpha}^{2}<0, \quad\left(\frac{\partial^{2} p_{\alpha}}{\partial \tau^{2}}\right)_{s}>0, \quad \alpha=L, R,
$$

for all $\rho>0$ and all $s$ in $\mathbb{R}$.

The systems $\mathcal{S}_{L}$ and $\mathcal{S}_{R}$ are strictly hyperbolic over the following set of states:

$$
\Omega=\left\{\mathbf{u} \in \mathbb{R}^{3}: \rho>0, u \in \mathbb{R}, \varepsilon>0\right\} .
$$

More precisely, for $\alpha=L, R$, the system $\mathcal{S}_{\alpha}$ admits the three eigenvalues,

$$
\lambda_{1}^{\alpha}(\mathbf{u})=u-c_{\alpha}, \quad \lambda_{2}^{\alpha}(\mathbf{u})=u, \quad \lambda_{3}^{\alpha}(\mathbf{u})=u+c_{\alpha},
$$

which are real and distinct. The characteristic field associated with $\lambda_{2}^{\alpha}$ is linearly degenerate, while the characteristic fields associated with $\lambda_{1}^{\alpha}$ and $\lambda_{3}^{\alpha}$ are genuinely nonlinear.

2.2. Coupling model. Following [4,5], and [34, we propose here to model the coupling between systems (2.1) thanks to a bounded vector-valued Dirac measure concentrated at the coupling interface $x=0$. Let us consider an application of the form $t \in \mathbb{R}_{+}^{*} \mapsto \mathcal{M}(t)=\left(\mathcal{M}_{\rho}(t), \mathcal{M}_{\rho u}(t), \mathcal{M}_{\rho E}(t)\right)^{T} \in \mathbb{R}^{3} ;$ we then look for a solution to the initial value problem

$$
\begin{aligned}
\partial_{t} \mathbf{u}+\partial_{x} \mathbf{f}(\mathbf{u}, x) & =\mathcal{M}(t) \delta_{x=0}, & & x \in \mathbb{R}, t>0, \\
\mathbf{u}(x, 0) & =\mathbf{u}_{0}(x), & & x \in \mathbb{R},
\end{aligned}
$$


where $\delta_{x=0}$ denotes the Dirac delta function. The components of $\mathcal{M}$ represent respectively the losses of mass, momentum and energy through the coupling interface. The discontinuous flux function introduced in (2.5a) is defined by

$$
\mathbf{f}(\mathbf{u}, x)= \begin{cases}\mathbf{f}_{L}(\mathbf{u}), & x<0 \\ \mathbf{f}_{R}(\mathbf{u}), & x>0\end{cases}
$$

where the flux depends on $x$ through the pressure law

$$
p(\tau, \varepsilon, x)= \begin{cases}p_{L}(\tau, \varepsilon), & x<0 \\ p_{R}(\tau, \varepsilon), & x>0\end{cases}
$$

Using standard arguments (see for instance [41]), any solution to the coupling problem (2.5) is subject to the following coupling conditions through the interface

$$
\mathbf{f}_{R}\left(\mathbf{u}\left(0^{+}, t\right)\right)-\mathbf{f}_{L}\left(\mathbf{u}\left(0^{-}, t\right)\right)=\mathcal{M}(t), \quad t>0,
$$

where $\mathbf{u}\left(0^{-}, t\right)$ and $\mathbf{u}\left(0^{+}, t\right)$ denote the left and right traces of the solution $\mathbf{u}$ at time $t$. This model allows the application of various coupling strategies. Indeed, the weight $\mathcal{M}(t)$ can be seen as a user parameter, whose definition results generally from physical motivations. For instance, the definition of a weight $\mathcal{M} \equiv 0$ allows us to ensure the conservation of $\mathbf{u}$ over the whole space domain. Conversely, a state coupling method with transmission of a set of variables may lead to the definition of a nonzero weight. Given an admissible change of variables $\mathbf{v}=\varphi_{\alpha}(\mathbf{u})$, $\alpha=L, R$, the state $\mathbf{v}$ can be transmitted through the coupling interface by setting $\varphi_{L}\left(\mathbf{u}\left(0^{-}, t\right)\right)=\varphi_{R}\left(\mathbf{u}\left(0^{+}, t\right)\right), t>0$ (see [8] for more details). For example, the transmission of primitive variables $\rho, u$ and $p$ through this interface results from $\varphi_{\alpha}(\rho, \rho u, \rho E):=\left(\rho, u, p_{\alpha}(\tau, \varepsilon)\right), \alpha=L, R$. The present contribution investigates the general case where all the weights may be nonzero.

Note that (2.5a) can be written equivalently as

$$
\left\{\begin{array}{l}
\partial_{t} \mathbf{u}+\partial_{x} \mathbf{F}(\mathbf{u}, H)-\mathcal{M}(t) \partial_{x} H=\mathbf{0}, \quad x \in \mathbb{R}, t>0, \\
\partial_{t} H=0
\end{array}\right.
$$

where $H=H_{x=0}$ is the Heaviside function and $\mathbf{F}(\mathbf{u}, H)=(1-H) \mathbf{f}_{L}(\mathbf{u})+H \mathbf{f}_{R}(\mathbf{u})$. Thus we get a nonconservative system in variables $(\mathbf{u}, H)$, for which the initial data is (2.5b) for $\mathbf{u}$ augmented by $H(x, 0)=H(x)$. The matrix of (2.8) writes

$$
M(\mathbf{u}, H)=\left(\begin{array}{cc}
\partial_{\mathbf{u}} \mathbf{F} & \mathbf{f}_{R}-\mathbf{f}_{L}-\mathcal{M}(t) \\
0 & 0
\end{array}\right)
$$

with $\partial_{\mathbf{u}} \mathbf{F}(\mathbf{u}, H)=(1-H) \mathbf{f}_{L}^{\prime}(\mathbf{u})+H \mathbf{f}_{R}^{\prime}(\mathbf{u})$. This shows clearly that the presence of the measure source terms adds to the initial waves, associated to the eigenvalues of $\mathbf{f}_{L / R}^{\prime}(\mathbf{u})$, a stationary discontinuity at $x=0$. The system is resonant if a genuinely nonlinear field (thus associated to an acoustic wave) superimposes this discontinuity, we will thus restrict ourselves to the study of subsonic flows; this assumption prevents this resonance phenomenon which brings nonuniqueness.

\section{Numerical MEthod}

The numerical method we present will eventually result in a classical algorithm which can be implemented as Section 4 proves. It is, however, interesting to understand the main elements which guided us to its derivation, since the scheme properties are obviously inherited from this construction. 
3.1. Relaxation approximation. We are interested in solving numerically (2.5). In the context of finite volume methods, the Godunov method requires the exact solution of Riemann problems associated to (2.5) and may be expensive because of the presence of nonlinearities due to the pressure law. The relaxation approach we will follow is an attempt to reduce the computational cost, still involving the Godunov method but on a simpler system approximating (2.5). It consists in the construction of a linear or quasilinear enlarged system, with only linearly degenerate fields, involving a source term which ensures that, in the regime of large values of a relaxation parameter $\lambda>0$, the solution of this system converges to the solution of the original system. We refer the reader to [17, 24, 55] for discussions on the relaxation approach in the approximation of hyperbolic systems of conservation laws, [16,53] for relaxation numerical methods in a general setting, or [9, 20, 22, 25, 27, 59, 60 for precise examples in the context of fluid flows.

Let us introduce the system

$$
\partial_{t} \mathbf{U}_{\lambda}+\partial_{x} \mathbf{F}\left(\mathbf{U}_{\lambda}\right)=\lambda \boldsymbol{\mathcal { R }}\left(\mathbf{U}_{\lambda}, x\right)+\widetilde{\mathcal{M}}(t) \delta_{x=0}, \quad x \in \mathbb{R}, t>0,
$$

with

$$
\mathbf{F}(\mathbf{U})=\left(\begin{array}{c}
\rho u \\
\rho u^{2}+\pi \\
\rho E u+\pi u \\
\rho \pi u+a^{2} u
\end{array}\right), \quad \mathcal{R}(\mathbf{U}, x)=\left(\begin{array}{c}
0 \\
0 \\
0 \\
\rho(p(\tau, \varepsilon, x)-\pi)
\end{array}\right), \quad \widetilde{\mathcal{M}}(t)=\left(\begin{array}{c}
\mathcal{M}(t) \\
\mathcal{M}_{\rho \pi}(t)
\end{array}\right)
$$

where $a>0$ is a given constant which will be specified below. We have introduced a new variable $\pi$ which can be seen as a linearization of the original pressure $p$ and is called the relaxed pressure; in the source term $\mathcal{R}$, the pressure $p$ is defined by (2.6). With some abuse of notation, we note $\mathbf{U}=(\mathbf{u}, \rho \pi)$ (the rigorous notation should be $\left.\mathbf{U}=\left(\mathbf{u}^{T}, \rho \pi\right)^{T}\right)$, the vector of variables $\mathbf{U}$ is assumed to be defined on the set of states $\Omega^{r}=\Omega \times \mathbb{R}$ and we introduce $\Pi$ as the projection operator $\Pi: \mathbb{R}^{4} \rightarrow \mathbb{R}^{3}$ such that $\Pi \mathbf{U}=\mathbf{u}$. Formally, we can observe that the projection $\Pi \mathbf{U}_{\lambda}$ of the solution of the relaxation system (3.1) converges to a solution of the original system (2.5) (this last system is thus called the equilibrium system) when the relaxation parameter $\lambda$ goes to infinity since the fourth equation in (3.1) imposes

$$
\lim _{\lambda \rightarrow \infty} \pi_{\lambda}=p,
$$

(at least formally).

The time dependent weight $\mathcal{M}_{\rho \pi}(t) \in \mathbb{R}$ corresponds to a new degree of freedom and will be defined later on.

For ease of notation, we now omit the subscript $\lambda$. The homogeneous system (3.1) with $\lambda=0$ and $\widetilde{\mathcal{M}} \equiv 0$ is hyperbolic on $\Omega^{r}$ and possesses the eigenvalues

$$
\lambda_{1}^{r}(\mathbf{U})=u-a \tau, \quad \lambda_{2}^{r}(\mathbf{U})=u, \quad \lambda_{3}^{r}(\mathbf{U})=u+a \tau,
$$

where the eigenvalue $\lambda_{2}^{r}$ is double. All the characteristic fields associated with these eigenvalues are linearly degenerate. As seen when writing system (2.8), the presence of the source term in (3.1) results in an additional stationary discontinuity, which, with some abuse, we also call a contact wave with speed $\lambda_{0}^{r}(\mathbf{U})=0$ located at the coupling interface. The coefficient $a$ has the dimension of a Lagrangian sound speed, and appears as a linearization of $\rho c$. In order to prevent system (3.1) from instabilities in the regime of large values of $\lambda$, this coefficient $a$ must verify the 
so-called subcharacteristic condition

$$
a>\max \left(\rho c_{\alpha}\right), \quad \alpha=L, R,
$$

for all the states under consideration. This condition expresses the interlacing of the eigenvalues of the relaxation and equilibrium systems $u-\tau a<u-c_{\alpha}<u<u+c_{\alpha}<$ $u+\tau a$. We refer the reader to [17] for a rigorous study of stability conditions.

Any solution to (3.1) with initial condition (and a finite relaxation rate $\lambda>0$ ), is subject to the following coupling condition through the coupling interface

$$
\mathbf{F}\left(\mathbf{U}\left(0^{+}, t\right)\right)-\mathbf{F}\left(\mathbf{U}\left(0^{-}, t\right)\right)=\widetilde{\mathcal{M}}(t), \quad t>0 .
$$

Finally, note that the flux $\mathbf{F}(\mathbf{U})$ in (3.1) is now globally defined and does not depend on the space variable, contrary to the flux $\mathbf{f}(\mathbf{u}, x)$ in (2.5) .

3.2. The main features of the scheme. The approach we follow involves an enlarged system (3.1) with two different source terms. The first one corresponds to relaxation and the second one to the original point source term. The items are linked in that the relaxation procedure is introduced as a practical mean to ensure the well-balanced property, i.e., to preserve the stationary solutions associated to the point source. However, the two source terms are not treated in the same way in the time discretization which involves a time splitting technique.

Let $\Delta t$ be a time step, and set $t^{(n)}=n \Delta t, n \geq 0$. Using a time splitting technique, the first step concerns an enlarged system, one solves (3.1) with $\lambda=0$ on an interval of length $\Delta t$, say $\left.] t^{(n)}, t^{(n+1)}\right]$, which allows solutions away from equilibrium but ensures the well-balanced property. Relaxation towards equilibrium is performed instantaneously in a second step, which amounts to solving

$$
\partial_{t} \mathbf{U}_{\lambda}=\lambda \boldsymbol{R}\left(\mathbf{U}_{\lambda}, x\right)
$$

on $\left.] t^{(n)}, t^{(n+1)}\right]$, starting with the data, noted $\mathbf{U}_{\lambda}\left(t^{(n+1)-}\right)$, given by the solution of the first step at time $t^{(n+1)}$, and let $\lambda \rightarrow \infty$. In the time discretization of this step, we use a simple implicit Euler method, so that $\mathbf{u}_{\lambda}\left(t^{(n+1)}\right)=\mathbf{u}_{\lambda}\left(t^{(n+1)-}\right)$ and as $\lambda \rightarrow \infty, \rho(p-\pi)\left(t^{(n+1)}, x\right) \rightarrow 0$.

For our fully discrete scheme, in which well-balanced and time stepping techniques complete each other, several ingredients are needed, they are described precisely in the following subsections. Let us make first two comments ((i) and (ii) below) because the similarity between the two source terms, similarity already pointed out in [45], may, however, introduce some confusion. Indeed, the treatment of the relaxation source term (in the limit $\lambda \rightarrow \infty$ ) is responsible for discontinuity in the approximate solution at each time $t^{(n+1)-}$. While the well-balanced property is achieved on the ground of a geometric source term taking the form of a Dirac measure in space. We detail these points in (ii). Moreover, both relaxation and point source terms involve some notions of equilibria. We must however distinguish the underlying notions which we do below:

(i) Concerning relaxation, the first notion of equilibrium refers to instantaneous relaxation. The formal limit, letting the relaxation time $\lambda^{-1}$ goes to 0 in (3.1), yields the equilibrium system (2.5). Associated to this latter system are the equilibria states $\mathbf{U}^{e q}=(\mathbf{u}, \rho p)$. This naturally defines an operator $\mathbf{U} \rightarrow \mathbf{U}^{e q}$ which we denote $\mathcal{E}$. This operator is involved in the second step of the splitting method and made precise in Section 3.4 . 


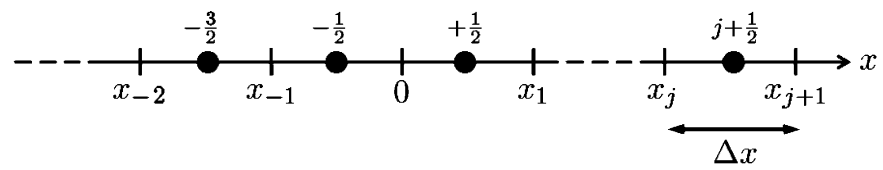

Figure 1. Space mesh.

The second notion of equilibrium refers to the well-balanced property, which is often defined as preserving equilibria. In this context, equilibria are thus stationary solutions of the PDE with point source (cf. Definition 3.1 below). They are analyzed in Section 3.5.

(ii) The treatment of the relaxation source term by a time splitting procedure is responsible for a discontinuity w.r.t. the time variable, for all $x \in \mathbb{R}$. Indeed, following L. Gosse in [46] (who refers to a prior work by F. Bouchut [15]), the classical time-splitting discretization of (3.1) leads to the equation

$$
\left.\partial_{t} \mathbf{U}+\partial_{x} \mathbf{F}(\mathbf{U})=\sum_{n>0}\left(\mathcal{E}\left(\mathbf{U}^{(n)-}\right)-\mathbf{U}^{(n)-}\right)\right) \delta_{t=t_{n}}+\widetilde{\mathcal{M}}(t) \delta_{x=0}, \quad x \in \mathbb{R}, t>0,
$$

where $\delta_{t=t^{(n)}}$ denotes the Dirac measure at point $t^{(n)}$, acting on functions of the time variable. We have noted $\mathbf{U}^{(n)-}=\mathbf{U}\left(., t^{(n)-}\right)$ the limit of $\mathbf{U}(., t)$ as $t \rightarrow t^{(n)}$, with $t<$ $t^{(n)}$. The added delta measure terms may be seen as coming from the discretization of the time variable, approximating $t$ by a step function equal to $t^{(n)}$ on $\left[t^{(n)}, t^{(n+1)}\right)$. This amounts to approximate $1=\partial_{t} t$ by a sum $\Delta t \sum_{n} \delta_{t=t(n)}$ of Dirac measures at points $t^{(n)}$ with weight $\Delta t$. This approximation mimicks the pioneering approach of [48] for the derivation of well-balanced schemes. Note that the approach in [48] considered an equation with a geometric source term, which thus concerns the space variable. Then, the discretization of the singular relaxation source term $\lambda \boldsymbol{R}(\mathbf{U})$ in the limit $\lambda \rightarrow \infty$ takes the form $\left.\sum_{n>0}\left(\mathcal{E}\left(\mathbf{U}^{(n)-}\right)-\mathbf{U}^{(n)-}\right)\right) \delta_{t=t_{n}}$. Formally, in the numerical treatment of (3.4), the relaxation time $\frac{1}{\lambda}$ is replaced by a time step $\Delta t$.

We now give the great lines of our fully discretized numerical method. In the above described time splitting algorithm, only the first step needs a space discretization. We use a Godunov type scheme with an exact Riemann solver for the enlarged system ( $(3.1)$ with $\lambda=0$ ) which is introduced in Section 3.3. Thus we need to solve the Riemann problem, which is performed in Section 3.6 (uniqueness requires we are away from resonance, a condition which is fulfilled by assuming that the flow is subsonic). We will see that adding the energy equation, while assuming nonzero weights, renders the computations trickier than in [5]. The relaxation procedure adds a weight $\mathcal{M}_{\rho \pi}$ which may be chosen (see Section 3.7) in order to preserve stationary solutions (defined in Section 3.5).

3.3. Godunov-type scheme. We aim at solving, numerically, the coupling problem (2.5) by a finite volume method, and first introduce some notations. Let $\Delta x$ be the space step which we take uniform for simplicity. The nodes of the spatial mesh are defined by $x_{j+\frac{1}{2}}=\frac{1}{2}\left(x_{j}+x_{j+1}\right), j \in \mathbb{Z}$ where the points $x_{j}=j \Delta x$ correspond to the interfaces between elements $\mathcal{C}_{j+\frac{1}{2}}=\left(x_{j}, x_{j+1}\right)$. The coupling interface is located in $x_{0}=0$ and the points $x_{j}$ are in the left (resp. right) domain if $j<0$ (resp. $j>0$ ) (see Figure 1). 
At each time step $t^{(n)}$, we look for a piecewise constant numerical solution $\mathbf{u}_{\Delta}\left(x, t^{(n)}\right)$ of the form

$$
\mathbf{u}_{\Delta}\left(x, t^{(n)}\right)=\mathbf{u}_{j+\frac{1}{2}}^{(n)}, \quad \forall x \in \mathcal{C}_{j+\frac{1}{2}} .
$$

For $t=0$, the initial condition reads

$$
\mathbf{u}_{j+\frac{1}{2}}^{(0)}=\frac{1}{\Delta x} \int_{\mathcal{C}_{j+\frac{1}{2}}} \mathbf{u}_{0}(x) d x, \quad \forall j \in \mathbb{Z} .
$$

For simplicity, we consider a three-point finite volume scheme of the form

$$
\begin{array}{ll}
\mathbf{u}_{j-\frac{1}{2}}^{(n+1)}=\mathbf{u}_{j-\frac{1}{2}}^{(n)}-\frac{\Delta t}{\Delta x}\left(\left(\mathbf{g}_{L}\right)_{j}^{n}-\left(\mathbf{g}_{L}\right)_{j-1}^{n}\right), & j \leq 0, \\
\mathbf{u}_{j+\frac{1}{2}}^{(n+1)}=\mathbf{u}_{j+\frac{1}{2}}^{(n)}-\frac{\Delta t}{\Delta x}\left(\left(\mathbf{g}_{R}\right)_{j+1}^{n}-\left(\mathbf{g}_{R}\right)_{j}^{n}\right), & j \geq 0,
\end{array}
$$

where the numerical flux

$$
\left(\mathbf{g}_{\alpha}\right)_{j}^{n}=\mathbf{g}_{\alpha}\left(\mathbf{u}_{j-\frac{1}{2}}^{(n)}, \mathbf{u}_{j+\frac{1}{2}}^{(n)}\right), \quad \alpha=L, R,
$$

corresponds to a consistent approximation of the exact flux $\mathbf{f}_{\alpha}$ and is obtained by an approximate Riemann solver. In order to construct this solver, we use the relaxation approach and consider system (3.1). At each time iteration, Riemann problems must be considered:

- a Riemann problem defined at any internal interface $x_{j}, j \neq 0$ :

$$
\left\{\begin{array}{l}
\partial_{t} \mathbf{U}+\partial_{x} \mathbf{F}(\mathbf{U})=0, \\
\mathbf{U}\left(x, t^{(n)}\right)= \begin{cases}\mathbf{U}_{j-\frac{1}{2}}^{(n)}, & x<x_{j}, \\
\mathbf{U}_{j+\frac{1}{2}}^{(n)}, & x>x_{j},\end{cases}
\end{array}\right.
$$

- a Riemann problem with source term at the coupling interface $x_{0}=0$ :

$$
\left\{\begin{aligned}
\partial_{t} \mathbf{U}+\partial_{x} \mathbf{F}(\mathbf{U}) & =\widetilde{\mathcal{M}}^{(n)} \delta_{x=0}, \quad \forall x \in\left(x_{-\frac{1}{2}}, x_{+\frac{1}{2}}\right), \quad t \in\left(t^{(n)}, t^{(n+1)}\right], \\
\mathbf{U}\left(x, t^{(n)}\right) & = \begin{cases}\mathbf{U}_{-\frac{1}{2}}^{(n)}, & x<0, \\
\mathbf{U}_{+\frac{1}{2}}^{(n)}, & x>0,\end{cases}
\end{aligned}\right.
$$

where $\widetilde{\mathcal{M}}^{(n)}$ denotes some consistent discretization of $\frac{1}{\Delta t} \int_{t^{(n)}}^{t^{(n+1)}} \widetilde{\mathcal{M}}(s) d s$, the average weight on $\left(t^{(n)}, t^{(n+1)}\right)$. In the above Riemann problems, for both cases we have defined the data $\mathbf{U}_{j \pm \frac{1}{2}}^{(n)}$ as

$$
\begin{aligned}
& \mathbf{U}_{j-\frac{1}{2}}^{(n)}=\left(\mathbf{u}_{j-\frac{1}{2}}^{(n)}, \rho_{j-\frac{1}{2}}^{(n)} p_{L}\left(\tau_{j-\frac{1}{2}}^{(n)}, \varepsilon_{j-\frac{1}{2}}^{(n)}\right)\right) \quad \text { if } \quad j \leq 0, \\
& \mathbf{U}_{j+\frac{1}{2}}^{(n)}=\left(\mathbf{u}_{j+\frac{1}{2}}^{(n)}, \rho_{j+\frac{1}{2}}^{(n)} p_{R}\left(\tau_{j+\frac{1}{2}}^{(n)}, \varepsilon_{j+\frac{1}{2}}^{(n)}\right)\right) \quad \text { if } \quad j \geq 0,
\end{aligned}
$$

which means we have completed the state $\mathbf{u}_{j \pm \frac{1}{2}}^{(n)}$ by a relaxed pressure at equilibrum, so that all the data $\mathbf{U}_{k+\frac{1}{2}}^{(n)}, k \in \mathbb{Z}$ are at equilibrium. 
3.4. Details of the time splitting method. In order to define the piecewise constant $\mathbf{u}_{\Delta}\left(x, t^{(n)}\right)=\left(\rho_{\Delta}, \rho u_{\Delta}, \rho E_{\Delta}\right)^{T}$ approximating the solution of (2.5) at time $t^{(n+1)}$, we proceed in two steps.

- First step: evolution in time $\left(t^{(n)} \rightarrow t^{(n+1)-}\right)$. We solve numerically the system (3.1) with $\lambda=0$. In this first step, we consider the Cauchy problem

$$
\left\{\begin{array}{llll}
\partial_{t} \mathbf{U}+\partial_{x} \mathbf{F}(\mathbf{U}) & =\widetilde{\mathcal{M}}^{(n)} \delta_{x=0}, & & x \in \mathbb{R}, \\
\mathbf{U}\left(x, t^{(n)}\right) & =\mathbf{U}_{\Delta}\left(x, t^{(n)}\right), & & x \in \mathbb{R},
\end{array}\right.
$$

with

$$
\mathbf{U}_{\Delta}\left(x, t^{(n)}\right)=\left(\mathbf{u}_{\Delta}\left(x, t^{(n)}\right),(\rho \pi)_{\Delta}\left(x, t^{(n)}\right)\right),
$$

and where $\rho \pi$ verifies the equilibrium condition

$$
(\rho \pi)_{\Delta}\left(x, t^{(n)}\right)=\rho_{\Delta}\left(x, t^{(n)}\right) p\left(\tau_{\Delta}\left(x, t^{(n)}\right), \varepsilon_{\Delta}\left(x, t^{(n)}\right), x\right) .
$$

The exact solution to (3.10) is obtained by juxtaposition of the solutions of the local Riemann problems set at each interface separating two adjacent elements. These solutions do not interact during the period $\Delta t$ if the space and time steps are linked by the usual Courant-Friedrich-Lewy condition:

$$
\frac{\Delta t}{\Delta x} \max _{\mathbf{U}}\left|\lambda_{i}^{r}(\mathbf{U})\right|<\frac{1}{2}, \quad i=0,1,2,3,
$$

where the maximum is taken over all the states $\mathbf{U}$ under consideration. We note that $\mathbf{U}\left(x, t^{(n+1)-}\right)$ is the solution obtained at the end of this step.

- Second step: instantaneous relaxation $\left(t^{(n+1)-} \rightarrow t^{(n+1)}\right)$. In this step, we solve the initial value problem

$$
\left\{\begin{array}{llll}
\partial_{t} \mathbf{U} & =\lambda \mathcal{R}(\mathbf{U}, x), & & x \in \mathbb{R}, \quad t \in\left(t^{(n)}, t^{(n+1)}\right], \\
\mathbf{U}\left(x, t^{(n)}\right)=\mathbf{U}_{\Delta}\left(x, t^{(n+1)-}\right), & & x \in \mathbb{R}, &
\end{array}\right.
$$

in the asymptotic regime $\lambda \rightarrow \infty$. This amounts to imposing that the function

$$
\mathbf{U}_{\Delta}\left(x, t^{(n+1)}\right)=\left(\mathbf{u}_{\Delta}\left(x, t^{(n+1)}\right),(\rho \pi)_{\Delta}\left(x, t^{(n+1)}\right)\right),
$$

has the following components:

$$
\begin{aligned}
\mathbf{u}_{\Delta}\left(x, t^{(n+1)}\right) & =\mathbf{u}_{\Delta}\left(x, t^{(n+1)-}\right), \\
(\rho \pi)_{\Delta}\left(x, t^{(n+1)}\right) & =\rho_{\Delta}\left(x, t^{(n+1)-}\right) p\left(\tau_{\Delta}\left(x, t^{(n+1)-}\right), \varepsilon_{\Delta}\left(x, t^{(n+1)-}\right), x\right) .
\end{aligned}
$$

As usual these two steps are followed by a projection of $\mathbf{u}_{\Delta}\left(x, t^{(n+1)}\right)$ on piecewise constant functions in order to define $\mathbf{u}_{j+\frac{1}{2}}^{(n+1)}$.

3.5. Equilibrium solutions. Following [34], given a weight

$$
\mathcal{M}=\left(\mathcal{M}_{\rho}, \mathcal{M}_{\rho u}, \mathcal{M}_{\rho E}\right)^{T},
$$

we look for a definition of the weight $\mathcal{M}_{\rho \pi}$ such that the above relaxation approach preserves the stationary solutions of the original coupling problem (2.5) in the sense of the following definition.

Definition 3.1. Let $\mathcal{M}=\left(\mathcal{M}_{\rho}, \mathcal{M}_{\rho u}, \mathcal{M}_{\rho E}\right)^{T}$ be a constant weight and $\mathbf{u}_{L}, \mathbf{u}_{R}$ two constant states belonging to the space of states $\Omega$ such that

$$
\mathbf{f}_{R}\left(\mathbf{u}_{R}\right)-\mathbf{f}_{L}\left(\mathbf{u}_{L}\right)=\mathcal{M} \text {. }
$$


Then, the function defined by

$$
\mathbf{u}(x, t)= \begin{cases}\mathbf{u}_{L}, & x<0, t>0 \\ \mathbf{u}_{R}, & x>0, t>0\end{cases}
$$

is called an equilibrium solution for the original coupling problem (2.5).

Let us note that the stationary solutions of the coupled Riemann problem are an important tool in the study of solutions to conservation laws with discontinuous flux since they play the role of constants in the classical situation of a smooth flux (see 11 and references therein) and can thus be used in Kruzhkov type entropies; they are called elementary solutions and the set of such states gives birth to the notion of germs. The theory for systems with discontinuous flux is not as complete. However, the above definition is a natural extension to systems with source terms (hence to the nonconservative problem (2.5)). We will require our scheme to be well balanced in the sense that it should preserve these equilibria (we refer to [21] for some general considerations on well-balanced simple schemes). For a conservative system with a continuous flux, constants are elementary solutions, a conservative scheme with consistent flux preserves constant states. One simply imposes that there is only one numerical flux at each interface, and that this flux coincides with the exact flux if the states on each side are equal.

3.6. Riemann problem associated to the relaxation system. The first step of the splitting procedure in $\$ 3.4$ requires the solution to the Riemann problem associated to the system (3.1) with $\lambda=0$. In this paper, we restrict ourselves to the study of subsonic flows. Given two subsonic constant states $\mathbf{U}_{L}$ and $\mathbf{U}_{R}$ in $\Omega^{r}$, we solve the following initial value problem,

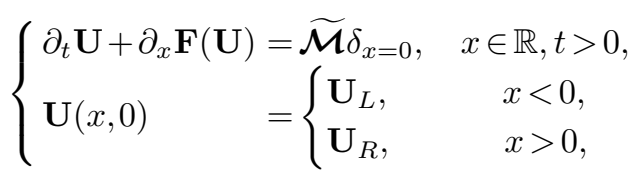

together with the coupling condition $(\underline{3.3})$, where $\widetilde{\mathcal{M}}$ does not depend on time to ensure self similarity in the solution.

The solution to (3.16) contains only contact discontinuities since all the fields associated with the eigenvalues of the system (3.1) (with $\lambda=0$ ) are linearly degenerate. Moreover, the Whitham condition (3.2) associated to the subsonic flow hypothesis lead us to consider two wave structures for the solution to (3.16). As shown in Figure 2, the intermediate states are denoted $\mathbf{U}_{L}, \mathbf{U}_{1}, \mathbf{U}_{-}, \mathbf{U}_{+}, \mathbf{U}_{R}$ when $\lambda_{2}^{r}<0$ and $\mathbf{U}_{L}, \mathbf{U}_{-}, \mathbf{U}_{+}, \mathbf{U}_{3}, \mathbf{U}_{R}$ when $\lambda_{2}^{r}>0$. In particular, we denote $\mathbf{U}_{ \pm}$the traces $\mathcal{W}\left(0 \pm ; \mathbf{U}_{L}, \mathbf{U}_{R}, \widetilde{\mathcal{M}}\right)$ at the interface $x=\lambda_{0}^{r}=0$. In order to respect these structures, the weights $\mathcal{M}_{\rho}, \mathcal{M}_{\rho u}, \mathcal{M}_{\rho E}$ and $\mathcal{M}_{\rho \pi}$ must be defined in an admissibility domain $\mathcal{D}_{a d m}\left(\mathbf{U}_{L}, \mathbf{U}_{R}\right)=\mathcal{D}_{a d m}^{-}\left(\mathbf{U}_{L}, \mathbf{U}_{R}\right) \cup \mathcal{D}_{a d m}^{+}\left(\mathbf{U}_{L}, \mathbf{U}_{R}\right)$. The definition of this domain is linked to the fact that, on the one hand, these weights must respect the subsonic structure of the solution and, on the other hand, the states are constrained to remain in the space of states $\Omega^{r}$. More precisely, using obvious notations for the components of the intermediate states, we define implicitly the admissible 


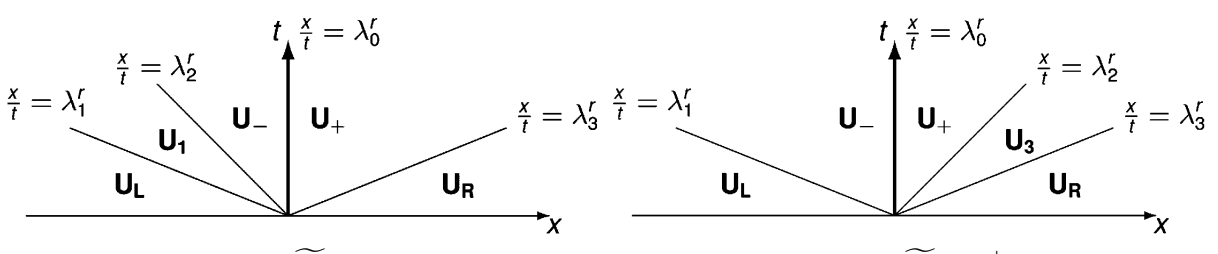

(a) Case $\widetilde{\mathcal{M}} \in \mathcal{D}_{\text {adm }}^{-}$.

(b) Case $\widetilde{\mathcal{M}} \in \mathcal{D}_{\text {adm }}^{+}$.

Figure 2. The two possible wave structures of the solution of the Riemann problem (3.16).

sets by

$$
\mathcal{D}_{a d m}^{-}=\left\{\widetilde{\mathcal{M}} \in \mathbb{R}^{4}:\left(\lambda_{1}^{r},-\tau_{1}, u_{-},-\tau_{-},-\tau_{+},-\lambda_{3}^{r},-\varepsilon_{1},-\varepsilon_{-},-\varepsilon_{+}\right)(\widetilde{\mathcal{M}})<0\right\},
$$

$$
\mathcal{D}_{a d m}^{+}=\left\{\widetilde{\mathcal{M}} \in \mathbb{R}^{4}:\left(\lambda_{1}^{r},-\tau_{3},-u_{+},-\tau_{-},-\tau_{+},-\lambda_{3}^{r},-\varepsilon_{3},-\varepsilon_{-},-\varepsilon_{+}\right)(\widetilde{\mathcal{M}})<0\right\} .
$$

Let us introduce the intermediate states

$$
\begin{array}{rlrl}
u^{*} & =\frac{1}{2}\left(u_{L}+u_{R}\right)-\frac{1}{2 a}\left(\pi_{R}-\pi_{L}\right), & \tau_{L}^{*}=\frac{1}{a}\left(u^{*}-\lambda_{1}^{r}\left(\mathbf{U}_{L}\right)\right), & \rho_{L}^{*}=\frac{1}{\tau_{L}^{*}}, \\
\pi^{*}=\frac{1}{2}\left(\pi_{L}+\pi_{R}\right)-\frac{a}{2}\left(u_{R}-u_{L}\right), & \tau_{R}^{*}=\frac{1}{a}\left(\lambda_{3}^{r}\left(\mathbf{U}_{R}\right)-u^{*}\right), & \rho_{R}^{*}=\frac{1}{\tau_{R}^{*}},
\end{array}
$$

and define the mass flows $m_{ \pm}:=\rho_{ \pm} u_{ \pm}$through the coupling interface. We also adopt the notation $w_{1}=u+\frac{\pi}{a}$ (resp. $w_{3}=u-\frac{\pi}{a}$ ), which is a 1- (resp. 3-) Riemann invariant in the case without measure (see [21]). Then, the following proposition gives the weak entropy self-similar solution to the Riemann problem (3.16).

Proposition 3.1. Let $\mathbf{U}_{L}$ and $\mathbf{U}_{R}$ in $\Omega^{r}$ be two subsonic constant states, and let $\mathcal{M}_{\rho}, \mathcal{M}_{\rho u}, \mathcal{M}_{\rho E}, \mathcal{M}_{\rho \pi}$ be the given source terms in $\mathcal{D}_{a d m}\left(\mathbf{U}_{L}, \mathbf{U}_{R}\right)$. Then, the Riemann problem (3.16) has a unique subsonic self-similar solution which we denote by $\mathcal{W}\left(\frac{x}{t} ; \mathbf{U}_{L}, \mathbf{U}_{R}, \widetilde{\mathcal{M}}\right)$. This function is defined by

$$
\mathcal{W}\left(\frac{x}{t} ; \mathbf{U}_{L}, \mathbf{U}_{R}, \widetilde{\mathcal{M}}\right)= \begin{cases}\mathbf{U}_{L}, & \frac{x}{t}<\lambda_{1}^{r}\left(\mathbf{U}_{L}\right), \\ \mathbf{U}_{1}, & \lambda_{1}^{r}\left(\mathbf{U}_{L}\right)<\frac{x}{t}<\lambda_{2}^{r}\left(\mathbf{U}_{1}\right), \\ \mathbf{U}_{-}, & \lambda_{2}^{r}\left(\mathbf{U}_{1}\right)<\frac{x}{t}<\lambda_{0}^{r}, \\ \mathbf{U}_{+}, & \lambda_{0}^{r}<\frac{x}{t}<\lambda_{3}^{r}\left(\mathbf{U}_{R}\right), \\ \mathbf{U}_{R}, & \frac{x}{t}>\lambda_{3}^{r}\left(\mathbf{U}_{R}\right),\end{cases}
$$

if $\widetilde{\mathcal{M}} \in \mathcal{D}_{\text {adm }}^{-}$and, respectively, by

$$
\mathcal{W}\left(\frac{x}{t} ; \mathbf{U}_{L}, \mathbf{U}_{R}, \widetilde{\mathcal{M}}\right)= \begin{cases}\mathbf{U}_{L}, & \frac{x}{t}<\lambda_{1}^{r}\left(\mathbf{U}_{L}\right), \\ \mathbf{U}_{-}, & \lambda_{1}^{r}\left(\mathbf{U}_{L}\right)<\frac{x}{t}<\lambda_{0}^{r}, \\ \mathbf{U}_{+}, & \lambda_{0}^{r}<\frac{x}{t}<\lambda_{2}^{r}\left(\mathbf{U}_{3}\right), \\ \mathbf{U}_{3}, & \lambda_{2}^{r}\left(\mathbf{U}_{3}\right)<\frac{x}{t}<\lambda_{3}^{r}\left(\mathbf{U}_{R}\right), \\ \mathbf{U}_{R}, & \frac{x}{t}>\lambda_{3}^{r}\left(\mathbf{U}_{R}\right),\end{cases}
$$


if $\widetilde{\mathcal{M}} \in \mathcal{D}_{\text {adm }}^{+}$. Moreover, in the case where $\widetilde{\mathcal{M}} \in \mathcal{D}_{\text {adm }}^{-}$, we have

$$
\begin{array}{ll}
m_{-}=\frac{2 a u^{*}+\mathcal{M}_{\rho u}+\mathcal{M}_{\rho \pi} / a-\left(w_{1 L}+2 a \tau_{R}^{*}\right) \mathcal{M}_{\rho}}{2 a \tau_{R}^{*}}, & (u, \pi)_{1}=(u, \pi)_{-}, \tau_{1}=\tau_{L}+\frac{u_{1}-u_{L}}{a}, \\
m_{+}=\frac{2 a u^{*}+\mathcal{M}_{\rho u}+\mathcal{M}_{\rho \pi} / a-w_{1 L} \mathcal{M}_{\rho}}{2 a \tau_{R}^{*}}, & E_{1}=\frac{a E_{L}+\pi_{L} u_{L}-\pi_{-} u_{-}}{a}, \\
E_{-}=\frac{m_{+} E_{+}+\pi_{+} u_{+}-\pi_{-} u_{-}-\mathcal{M}_{\rho E}}{m_{-}}, & E_{+}=\frac{a E_{R}+\pi_{+} u_{+}-\pi_{R} u_{R}}{a},
\end{array}
$$

and in the case where $\widetilde{\mathcal{M}} \in \mathcal{D}_{\text {adm }}^{+}$

$$
\begin{array}{ll}
m_{-}=\frac{2 a u^{*}+\mathcal{M}_{\rho u}-\mathcal{M}_{\rho \pi} / a-w_{3 R} \mathcal{M}_{\rho}}{2 a \tau_{L}^{*}}, & E_{3}=\frac{a E_{R}+\pi_{+} u_{+}-\pi_{R} u_{R}}{a}, \\
m_{+}=\frac{2 a u^{*}+\mathcal{M}_{\rho u}-\mathcal{M}_{\rho \pi} / a-\left(w_{3 R}-2 a \tau_{L}^{*}\right) \mathcal{M}_{\rho}}{2 a \tau_{L}^{*}}, & (u, \pi)_{3}=(u, \pi)_{+}, \tau_{3}=\tau_{R}+\frac{u_{R}-u_{3}}{a}, \\
E_{+}=\frac{m_{-} E_{-}+\pi_{-} u_{-}-\pi_{+} u_{+}+\mathcal{M}_{\rho E}}{m_{+}}, & E_{-}=\frac{a E_{L}+\pi_{L} u_{L}-\pi_{-} u_{-}}{a} .
\end{array}
$$

Finally, the traces of the solution are given by the relations

$$
\begin{aligned}
& u_{-}=u^{*}+\frac{a \mathcal{M}_{\rho u}-\mathcal{M}_{\rho \pi}-\left(a u_{R}-\pi_{R}\right) \mathcal{M}_{\rho}}{2 a\left(a-m_{-}\right)}, u_{+}=u^{*}+\frac{a \mathcal{M}_{\rho u}+\mathcal{M}_{\rho \pi}-\left(\pi_{L}+a u_{L}\right) \mathcal{M}_{\rho}}{2 a\left(a+m_{+}\right)}, \\
& \pi_{-}=\pi^{*}-\frac{a \mathcal{M}_{\rho u}-\mathcal{M}_{\rho \pi}+\left(\pi_{R}-a u_{R}\right) \mathcal{M}_{\rho}}{2\left(a-m_{-}\right)}, \pi_{+}=\pi^{*}+\frac{a \mathcal{M}_{\rho u}+\mathcal{M}_{\rho \pi}-\left(\pi_{L}+a u_{L}\right) \mathcal{M}_{\rho}}{2\left(a+m_{+}\right)},
\end{aligned}
$$

together with $\rho_{-}=\frac{m_{-}}{u_{-}}, \rho_{+}=\frac{m_{+}}{u_{+}}$.

Proof. We only consider the case $\widetilde{\mathcal{M}} \in \mathcal{D}_{\text {adm }}^{-}$, the case $\widetilde{\mathcal{M}} \in \mathcal{D}_{\text {adm }}^{+}$being symmetric. Recall that all characteristic fields are linearly degenerate so that only contact discontinuities are involved.

The continuity of the wave speeds for the $\lambda_{1-}^{r}, \lambda_{2^{-}}^{r}$ and $\lambda_{3}^{r}$-waves reads

$$
\begin{aligned}
\tau_{1} & =\tau_{L}+\frac{u_{1}-u_{L}}{a}, \\
u_{1} & =u_{-}, \\
u_{R}+\frac{a}{\rho_{R}} & =u_{+}+\frac{a}{\rho_{+}} .
\end{aligned}
$$

Through the steady interface $\lambda_{0}^{r}=0$, the relations (3.3) read

$$
\begin{aligned}
\mathcal{M}_{\rho} & =m_{+}-m_{-}, \\
\mathcal{M}_{\rho u} & =m_{+} u_{+}+\pi_{+}-m_{-} u_{-}-\pi_{-}, \\
\mathcal{M}_{\rho E} & =m_{+} E_{+}+\pi_{+} u_{+}-m_{-} E_{-}-\pi_{-} u_{-}, \\
\mathcal{M}_{\rho \pi} & =m_{+} \pi_{+}+a^{2} u_{+}-m_{-} \pi_{-}-a^{2} u_{-} .
\end{aligned}
$$

Across the $\lambda_{1}^{r}$-wave, the Rankine-Hugoniot relations on the momentum and energy balance equations read

$$
\left\{\begin{array}{l}
\lambda_{1}^{r}\left(\mathbf{U}_{L}\right)\left(\rho_{1} u_{1}-\rho_{L} u_{L}\right)=\rho_{1} u_{1}^{2}+\pi_{1}-\rho_{L} u_{L}^{2}-\pi_{L}, \\
\lambda_{1}^{r}\left(\mathbf{U}_{L}\right)\left(\rho_{1} E_{1}-\rho_{L} E_{L}\right)=\rho_{1} E_{1} u_{1}+\pi_{1} u_{1}-\rho_{L} E_{L} u_{L}-\pi_{L} u_{L},
\end{array}\right.
$$


which implies

$$
\begin{aligned}
\pi_{1}-\pi_{L}-a u_{L}+a u_{1} & =0, \\
E_{1}-E_{L}-\frac{1}{a}\left(\pi_{L} u_{L}-\pi_{1} u_{1}\right) & =0 .
\end{aligned}
$$

Considering now the $\lambda_{2}^{r}$-wave and the Rankine-Hugoniot relations applied to the energy balance equation, we have

$$
\lambda_{2}^{r}\left(\mathbf{U}_{1}\right)\left(\rho_{-} E_{-}-\rho_{1} E_{1}\right)=\rho_{-} E_{-} u_{-}+\pi_{-} u_{-}-\rho_{1} E_{1} u_{1}-\pi_{1} u_{1},
$$

and using (3.19b), we obtain

$$
\pi_{1}=\pi_{-} .
$$

From (3.19b) and (3.22), we obtain that the equation (3.21a is equivalent to

$$
\pi_{-}-\pi_{L}-a u_{L}+a u_{-}=0,
$$

which means $w_{1 L}=w_{1-}$. Next, through the $\lambda_{3}^{r}$-contact, the Rankine-Hugoniot relations on the momentum and energy balance equations read

$$
\left\{\begin{array}{l}
\lambda_{3}^{r}\left(\mathbf{U}_{+}\right)\left(\rho_{R} u_{R}-\rho_{+} u_{+}\right)=\rho_{R} u_{R}^{2}+\pi_{R}-\rho_{+} u_{+}^{2}-\pi_{+}, \\
\lambda_{3}^{r}\left(\mathbf{U}_{+}\right)\left(\rho_{R} E_{R}-\rho_{+} E_{+}\right)=\rho_{R} E_{R} u_{R}+\pi_{R} u_{R}-\rho_{+} E_{+} u_{+}-\pi_{+} u_{+},
\end{array}\right.
$$

and it follows that

$$
\begin{aligned}
a u_{R}-a u_{+} & =\pi_{R}-\pi_{+}, \text {or } w_{3+}=w_{3 R}, \\
a E_{R}-a E_{+} & =\pi_{R} u_{R}-\pi_{+} u_{+} .
\end{aligned}
$$

The traces $u_{-}, u_{+}, \pi_{-}, \pi_{+}$are then obtained by solving the linear system of equations (3.20b), (3.20d), (3.23) and (3.24a), which is left to the reader.

From relations (3.24b) and (3.20c), we get

$$
E_{+}=\frac{a E_{R}+\pi_{+} u_{+}-\pi_{R} u_{R}}{a},
$$

and

$$
E_{-}=\frac{m_{+} E_{+}+\pi_{+} u_{+}-\pi_{-} u_{-}-\mathcal{M}_{\rho E}}{m_{-}} .
$$

Next, we determine the expressions of $m_{+}$and $m_{-}$. The expression of the state $u_{+}$ gives

$$
2 a\left(a+m_{+}\right)\left(u_{+}-u^{*}\right)=a \mathcal{M}_{\rho u}+\mathcal{M}_{\rho \pi}-\left(a u^{*}+\pi^{*}\right) \mathcal{M}_{\rho},
$$

then, multiplying the relation (3.19c) by $\rho_{+}$, we have

$$
m_{+}+a=\rho_{+}\left(u_{R}+\frac{a}{\rho_{R}}\right),
$$

so that

$$
\left(a+m_{+}\right) u_{+}=m_{+}\left(u_{R}+\frac{a}{\rho_{R}}\right) \text {, }
$$

and

$$
\begin{aligned}
2 a\left(a+m_{+}\right)\left(u_{+}-u^{*}\right) & =2 a m_{+}\left(u_{R}+\frac{a}{\rho_{R}}\right)-2 a^{2} u^{*}-2 a m_{+} u^{*} \\
& =\frac{2 a^{2}}{\rho_{R}^{*}} m_{+}-2 a^{2} u^{*} .
\end{aligned}
$$


Moreover, from (3.27), we obtain

$$
m_{+}=\frac{2 a^{2} u^{*}+a \mathcal{M}_{\rho u}+\mathcal{M}_{\rho \pi}-\left(\pi_{L}+a u_{L}\right) \mathcal{M}_{\rho}}{2 a^{2} \tau_{R}^{*}},
$$

and from (3.20a we finally get

$$
m_{-}=\frac{2 a^{2} u^{*}+a \mathcal{M}_{\rho u}+\mathcal{M}_{\rho \pi}-\left(\pi_{L}+a u_{L}+2 a^{2} \tau_{R}^{*}\right) \mathcal{M}_{\rho}}{2 a^{2} \tau_{R}^{*}},
$$

which ends the proof of the case under consideration.

We check that we do recover the formulas of [5] in the case $\mathcal{M}_{\rho}=0$. Now, we can define the numerical fluxes (3.6)-(3.7) introduced in $\$ 3.3$. Recall that we have denoted $\Pi$ the projection operator defined as $\Pi:\left(x_{1}, x_{2}, x_{3}, x_{4}\right) \in \mathbb{R}^{4} \mapsto\left(x_{1}, x_{2}, x_{3}\right) \in$ $\mathbb{R}^{3}$. Then, the numerical fluxes $\left(\mathbf{g}_{L}\right)_{j}^{n}$ and $\left(\mathbf{g}_{R}\right)_{j}^{n}$ are given by the following Godunov type numerical fluxes:

$$
\begin{aligned}
& \left(\mathbf{g}_{L}\right)_{j}^{n}=\Pi \mathbf{F}\left(\mathcal{W}\left(0^{-} ; \mathbf{U}_{j-\frac{1}{2}}^{(n)}, \mathbf{U}_{j+\frac{1}{2}}^{(n)}, \widetilde{\mathcal{M}}_{j}^{(n)}\right)\right), \quad j \leq 0, \\
& \left(\mathbf{g}_{R}\right)_{j}^{n}=\Pi \mathbf{F}\left(\mathcal{W}\left(0^{+} ; \mathbf{U}_{j-\frac{1}{2}}^{(n)}, \mathbf{U}_{j+\frac{1}{2}}^{(n)}, \widetilde{\mathcal{M}}_{j}^{(n)}\right)\right), \quad j \geq 0,
\end{aligned}
$$

where the weights $\widetilde{\mathcal{M}}_{j}^{(n)}$ are defined by

$$
\widetilde{\mathcal{M}}_{j}^{(n)}=\left\{\begin{array}{lll}
\mathbf{0}, & \text { if } & j \neq 0, \\
\widetilde{\mathcal{M}}^{(n)}, & \text { if } & j=0 .
\end{array}\right.
$$

Note that for $j \neq 0$, the solution $\mathcal{W}$ of the Riemann problem may be discontinuous at 0 but the flux $\mathbf{F}$ is continuous (see indeed (3.3)) so that the choice to take the value of $\mathcal{W}\left(0^{ \pm}\right)$is not important; the numerical flux is just the projection of the exact Godunov flux for the relaxation system. While, for $j=0$, there are two fluxes linked by the discrete coupling condition (discrete version of (2.7))

$$
\left(\mathbf{g}_{R}\right)_{0}^{n}-\left(\mathbf{g}_{L}\right)_{0}^{n}=\mathcal{M}^{(n)} .
$$

This situation with two fluxes at an interface is now classical for finite volume schemes approximating a system with geometric source terms.

3.7. Preserving equilibria. Next, it remains to determine the additional weight $\mathcal{M}_{\rho \pi}$ such that the relaxation approach preserves the equilibrium solutions of the original coupled problem (2.5) (see Definition 3.1). The following proposition gives this weight.

Proposition 3.2. Let $\mathbf{u}_{L}$ and $\mathbf{u}_{R}$ be two subsonic constant states belonging to $\Omega$ and a weight $\mathcal{M}=\left(\mathcal{M}_{\rho}, \mathcal{M}_{\rho u}, \mathcal{M}_{\rho E}\right)^{T}$ such that the relation (3.15) holds true. Let us define the mass flows through the interface $m_{ \pm}^{e}=m_{ \pm}^{e}\left(\mathbf{u}_{L}, \mathbf{u}_{R}, \mathcal{M}_{\rho}, \mathcal{M}_{\rho u}\right)$ as

$$
\begin{aligned}
& m_{+}^{e}:=\frac{\mathcal{M}_{\rho u}+2 a u^{*}+\left(a \tau_{L}^{*}-u^{*}\right) \mathcal{M}_{\rho}}{a\left(\tau_{L}^{*}+\tau_{R}^{*}\right)}, \\
& m_{-}^{e}:=m_{+}^{e}-\mathcal{M}_{\rho},
\end{aligned}
$$

together with the weight $\mathcal{M}_{\rho \pi}^{e}=\mathcal{M}_{\rho \pi}^{e}\left(\mathbf{u}_{L}, \mathbf{u}_{R}, \mathcal{M}_{\rho}, \mathcal{M}_{\rho u}\right)$, given by

$$
\mathcal{M}_{\rho \pi}^{e}:=\mathscr{P}_{R} m_{+}^{e}-\mathscr{P}_{L} m_{-}^{e},
$$


where $\mathscr{P}_{\alpha}=p_{\alpha}\left(\tau_{\alpha}, \varepsilon_{\alpha}\right)+a^{2} \tau_{\alpha}$, with $\alpha=L, R$. Then, the relaxation method in Proposition 3.1 with the weight $\widetilde{\mathcal{M}}^{e}=\left(\mathcal{M}_{\rho}, \mathcal{M}_{\rho u}, \mathcal{M}_{\rho E}, \mathcal{M}_{\rho \pi}^{e}\right)^{T}$ preserves the equilibrium solutions of the coupled problem (2.5).

Proof. Let $\mathbf{u}_{L}, \mathbf{u}_{R}$ in $\Omega$ be such that the relation (3.15) holds true and assume that $m_{+}^{e}, m_{-}^{e}, \mathscr{P}_{R}, \mathscr{P}_{L}$, and $\mathcal{M}_{\rho \pi}^{e}$ satisfy relations (3.30) to (3.32). Let us show that

$$
\mathcal{W}\left(\frac{x}{t} ; \mathbf{U}_{L}, \mathbf{U}_{R}, \widetilde{\mathcal{M}}^{e}\right)= \begin{cases}\mathbf{U}_{L}, & x<0 \\ \mathbf{U}_{R}, & x>0\end{cases}
$$

where $\mathbf{U}_{\alpha}=\left(\mathbf{u}_{\alpha}, \rho_{\alpha} p_{\alpha}\right), \alpha=L, R$ and where $\mathcal{W}$ is the solution to the Riemann problem (3.16) solved explicitly in Proposition 3.1. Therefore, we must prove that

$$
\left\{\begin{array}{lllll}
\mathbf{U}_{-}=\mathbf{U}_{1}=\mathbf{U}_{L} & \text { and } & \mathbf{U}_{+}=\mathbf{U}_{R} & \text { if } & \widetilde{\mathcal{M}}_{e}^{e} \in \mathcal{D}_{a d m}^{-} \\
\mathbf{U}_{+}=\mathbf{U}_{3}=\mathbf{U}_{R} & \text { and } & \mathbf{U}_{-}=\mathbf{U}_{L} & \text { if } & \widetilde{\mathcal{M}}_{e} \in \mathcal{D}_{a d m}^{+}
\end{array}\right.
$$

Here we present only the evaluation of the trace state $\mathbf{U}_{+}$since the approach is similar for the other states.

By Proposition 3.1, note that the mass flows $m_{ \pm}^{e}$ are identical for the two considered wave structures. Let $m_{\alpha}=\rho_{\alpha} u_{\alpha}, \alpha=L, R$. From (3.15), we have

$$
\begin{aligned}
\mathcal{M}_{\rho} & =m_{R}-m_{L} \\
\mathcal{M}_{\rho u} & =m_{R} u_{R}+p_{R}-m_{L} u_{L}-p_{L} \\
\mathcal{M}_{\rho E} & =m_{R} E_{R}+p_{R} u_{R}-m_{L} E_{L}-p_{L} u_{L}
\end{aligned}
$$

By plugging the weights $\mathcal{M}_{\rho}$ and $\mathcal{M}_{\rho u}$ into (3.30), and using the relations $a \tau_{L}^{*}-u^{*}=$ $a \tau_{L}-u_{L}$ and $\pi_{\alpha}=p_{\alpha}$ with $\alpha=L, R$, we obtain

$$
m_{+}^{e}=\frac{m_{R}\left(a\left(\tau_{L}+\tau_{R}\right)+u_{R}-u_{L}\right)}{a\left(\tau_{L}^{*}+\tau_{R}^{*}\right)} .
$$

From the definition of the intermediate states $\tau_{L}^{*}$ and $\tau_{R}^{*}$, we have

$$
a\left(\tau_{L}^{*}+\tau_{R}^{*}\right)=\lambda_{3}\left(\mathbf{U}_{R}\right)-\lambda_{1}\left(\mathbf{U}_{L}\right)=a\left(\tau_{L}+\tau_{R}\right)+u_{R}-u_{L}
$$

and

$$
m_{+}^{e}=m_{R}
$$

Using relation (3.34a), it follows that

$$
m_{-}^{e}=m_{+}^{e}-\mathcal{M}_{\rho}=m_{R}-\left(m_{R}-m_{L}\right)=m_{L}
$$

Let us now consider the state $u_{+}$. From Proposition 3.1, the equalities $\pi_{\alpha}=p_{\alpha}$ with $\alpha=L, R$, the definitions of the intermediate state $u^{*}$ and the weight $\mathcal{M}_{\rho \pi}^{e}(\underline{3.32})$, 
together with the relations (3.34a), (3.34b), (3.35) and (3.36), we get

$$
\begin{aligned}
u_{+}= & u^{*}+\frac{a \mathcal{M}_{\rho u}+\mathcal{M}_{\rho \pi}^{e}-\left(\pi_{L}+a u_{L}\right) \mathcal{M}_{\rho}}{2 a\left(a+m_{+}^{e}\right)} \\
= & \frac{u_{R}+u_{L}}{2}+\frac{p_{L}-p_{R}}{2 a}+\frac{a\left(m_{R} u_{R}+p_{R}-m_{L} u_{L}-p_{L}\right)+\left(p_{R}+a^{2} \tau_{R}\right) m_{R}}{2 a\left(a+m_{R}\right)} \\
& \quad-\frac{\left(p_{L}+a^{2} \tau_{L}\right) m_{L}}{2 a\left(a+m_{R}\right)}-\frac{\left(p_{L}+a u_{L}\right)\left(m_{R}-m_{L}\right)}{2 a\left(a+m_{R}\right)} \\
= & \frac{u_{R}+u_{L}}{2}+\frac{p_{L}-p_{R}}{2 a}+\frac{a u_{R}\left(m_{R}+a\right)+p_{R}\left(a+m_{R}\right)-\left(p_{L}+a u_{L}\right)\left(a+m_{R}\right)}{2 a\left(a+m_{R}\right)} \\
= & u_{R},
\end{aligned}
$$

and it follows immediately that $\rho_{+}=m_{+}^{e} / u_{+}=m_{R} / u_{R}=\rho_{R}$. Next, using similar arguments together with the definition of the state $\pi^{*}$, we obtain

$$
\begin{aligned}
\pi_{+} & =\pi^{*}+\frac{a \mathcal{M}_{\rho u}+\mathcal{M}_{\rho \pi}^{e}-\left(\pi_{L}+a u_{L}\right) \mathcal{M}_{\rho}}{2\left(a+m_{+}^{e}\right)} \\
& =\frac{\pi_{L}+\pi_{R}}{2}+\frac{a\left(u_{L}-u_{R}\right)}{2}+\frac{\left(a+m_{R}\right)\left(a u_{R}+p_{R}-p_{L}-a u_{L}\right)}{2\left(a+m_{R}\right)} \\
& =\frac{p_{L}+p_{R}}{2}+\frac{a\left(u_{L}-u_{R}\right)}{2}+\frac{p_{R}-p_{L}+a\left(u_{R}-u_{L}\right)}{2} \\
& =p_{R} .
\end{aligned}
$$

Now, for the evaluation of $E_{+}$, two cases must be considered. If $\widetilde{\mathcal{M}}^{e} \in \mathcal{D}_{a d m}^{-}$, we use Proposition 3.1 and the equality $\pi_{+} u_{+}=\pi_{R} u_{R}$, and immediately get

$$
E_{+}=\frac{a E_{R}+\pi_{+} u_{+}-\pi_{R} u_{R}}{a}=E_{R}
$$

On the other hand, if $\widetilde{\mathcal{M}}^{e} \in \mathcal{D}_{a d m}^{+}$, the energy is given by

$$
E_{+}=\frac{m_{-} E_{-}+\pi_{-} u_{-}-\pi_{+} u_{+}+\mathcal{M}_{\rho E}}{m_{+}} .
$$

Moreover, a computation similar to those made for the states $u_{+}$and $\pi_{+}$gives $\pi_{-} u_{-}=\pi_{L} u_{L}$ so that $E_{-}=E_{L}$ (cf. Proposition 3.1). Using these two last relations with equation (3.34c), we finally obtain

$$
E_{+}=\frac{m_{L} E_{L}+p_{L} u_{L}-p_{R} u_{R}+m_{R} E_{R}+p_{R} u_{R}-m_{L} E_{L}-p_{L} u_{L}}{m_{R}}=E_{R},
$$

which ends the proof.

3.8. Choices of the measure source terms. The measure source terms can be determined in a dynamic way, at each time step of a discrete time algorithm. We present different choices according to the chosen objective.

3.8.1. Flux coupling and state coupling. In model adaptation, one couples two different models at an interface which is fixed during a time interval of length the time step, but may vary at each time step. The position of the interface is optimized in such a way that in the region where one computes the coarse model, the model error between the fine and coarse models (evaluated by some indicator) is less than some given tolerance. Then, the coupling condition should not have a great impact, 
and one chooses at each time step the same, either a conservative (flux coupling) or a nonconservative (state coupling) approach [56.

At time $t^{(n)}$, the coupling condition (2.7) at the discrete level reads

$$
\mathcal{M}^{(n)}=\left(\mathbf{g}_{R}\right)_{0}^{n}-\left(\mathbf{g}_{L}\right)_{0}^{n} .
$$

The choice of a flux coupling leads to $\mathcal{M}^{(n)}=\mathbf{0}$ for all $n$ in $\mathbb{N}$. In order to ensure the state coupling we use an approach based on the introduction of reconstructed states. This procedure was proposed in [6], formalized in [8] and then adapted in the context of coupling with source terms [34. The method allows us to impose continuity (at the coupling interface) of a given set of variables. This can be related to other nonconservative approaches as the single fluid algorithm introduced in [1] and also to well-balanced schemes in the spirit of [44,48]; see [47.

The numerical fluxes $\left(\mathbf{g}_{L}\right)_{0}^{n}$ and $\left(\mathbf{g}_{R}\right)_{0}^{n}$ are given by

$$
\left(\mathbf{g}_{L}\right)_{0}^{n}=\mathbf{g}_{L}\left(\mathbf{u}_{-\frac{1}{2}}^{(n)}, \overline{\mathbf{u}}_{+\frac{1}{2}}^{(n)}\right), \quad\left(\mathbf{g}_{R}\right)_{0}^{n}=\mathbf{g}_{R}\left(\overline{\mathbf{u}}_{-\frac{1}{2}}^{(n)}, \mathbf{u}_{+\frac{1}{2}}^{(n)}\right),
$$

where (using the notations introduced in Section 2.2), the reconstructed states $\overline{\mathbf{u}}_{-\frac{1}{2}}^{(n)}$ and $\overline{\mathbf{u}}_{+\frac{1}{2}}^{(n)}$ are defined as

$$
\overline{\mathbf{u}}_{-\frac{1}{2}}^{(n)}:=\varphi_{R}^{-1} \circ \varphi_{L}\left(\mathbf{u}_{-\frac{1}{2}}^{(n)}\right), \quad \overline{\mathbf{u}}_{+\frac{1}{2}}^{(n)}:=\varphi_{L}^{-1} \circ \varphi_{R}\left(\mathbf{u}_{+\frac{1}{2}}^{(n)}\right) .
$$

We refer the reader to [6, 34] for examples of changes of variables.

3.8.2. Dynamic evaluation: constrained optimization problems. The aim of this section is the evaluation of an optimal source term relative to some cost function. At each time step, given a convex cost function $\mathcal{J}$ and a nonempty convex subset $\mathcal{D} \subset \mathcal{D}_{a d m}$, the problem consists in finding an optimal mass $\widetilde{\mathcal{M}}_{\text {opt }}^{e}$ in $\mathcal{D}$ such that

$$
\mathcal{J}\left(\widetilde{\mathcal{M}}_{\text {opt }}^{e}\right) \leq \mathcal{J}\left(\widetilde{\mathcal{M}}^{e}\right), \quad \forall \widetilde{\mathcal{M}}^{e} \in \mathcal{D}
$$

The objective function and the space of constraints are defined by physical motivations; for instance, in order to impose a mass flow on one side of the interface, or else to impose a cooling. The cost function is then constructed in relation to the objective (see 4.2 for several examples). At each time step, when this optimization problem is solved, we set $\mathcal{M}^{(n)}=\Pi \widetilde{\mathcal{M}}_{\text {opt }}^{e}$, where $\Pi$ is the projection operator introduced in 33.6 .

\section{Numerical EXPERIMENTS}

We use a computational domain $(-0.5,0.5)$ with 200 elements. The coupling interface is at $x=0$. Moreover, the final time of the simulation is $T=0.12$ and the ratios of specific heats are $\gamma_{L}=1.4$ and $\gamma_{R}=1.28$. In order to preserve equilibrium solutions of the problem, we impose $\mathcal{M}_{\rho \pi}=\mathcal{M}_{\rho \pi}^{e}$, where $\mathcal{M}_{\rho \pi}^{e}$ is defined by (3.32). The following test cases are based on Riemann problems where the discontinuity is located at $x=0$ and the different initial data are given in Table 1. Numerical experiments using state coupling are based on the continuity of primitive variables $\varphi_{\alpha}(\mathbf{u})=\left(\rho, u, p_{\alpha}\right), \alpha=L, R$. 
TABLE 1. Initial conditions for different test cases.

\begin{tabular}{|c||ccc|ccc|}
\hline Test case & $\rho_{L}$ & $u_{L}$ & $p_{L}$ & $\rho_{R}$ & $u_{R}$ & $p_{R}$ \\
\hline 1 & 1.6 & 0.4 & 2.35 & 1.6 & 0.4 & 2.35 \\
$2,3,4,5$ & 1.6 & 0.4 & 2.35 & 1.4 & 0.4 & 1.9 \\
\hline
\end{tabular}

4.1. Flux coupling vs. state coupling. Test case 1. In this first test case, we consider uniform initial data given in Table 1 . The density, velocity, pressure and internal energy profiles are displayed in Figure 3 when applying flux coupling and state coupling in primitive variables $(\rho, u, p)$. Figure 4 displays the relative conservation losses in mass, momentum and total energy, obtained by integrating the conservation laws over space and time.

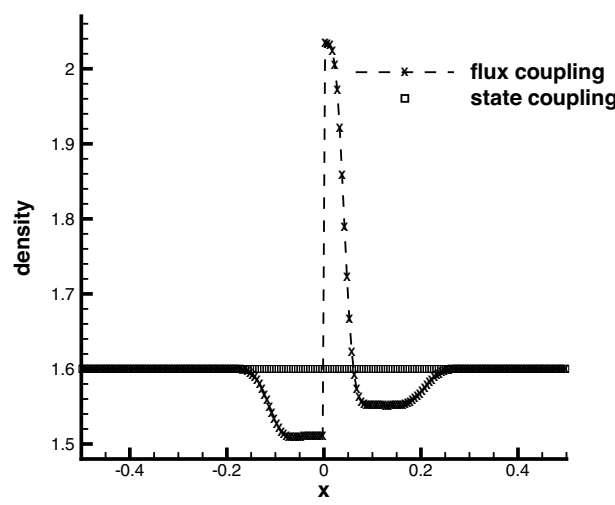

(a)

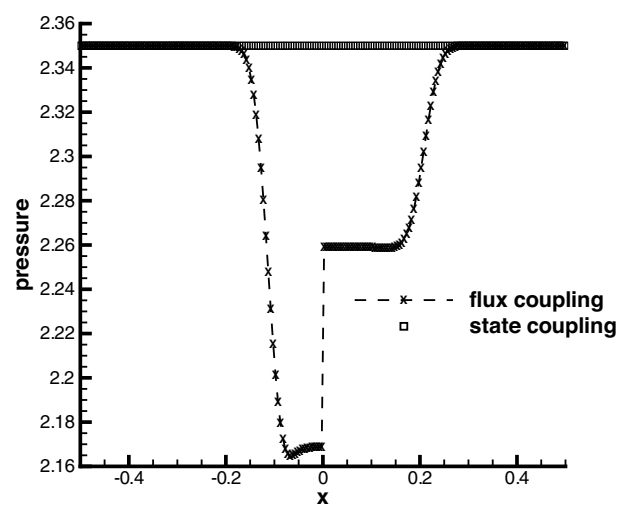

(c)

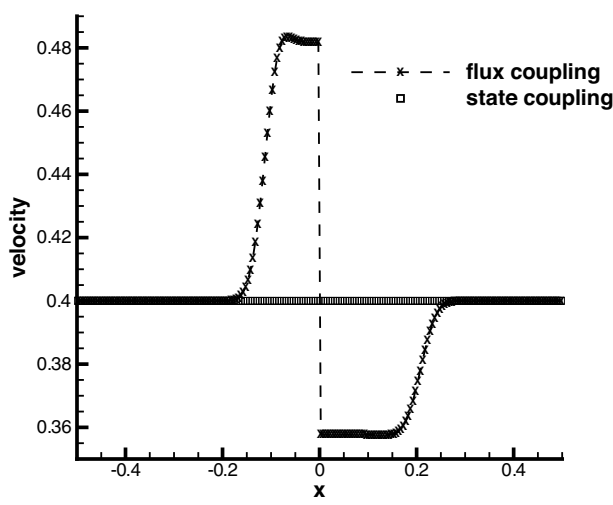

(b)

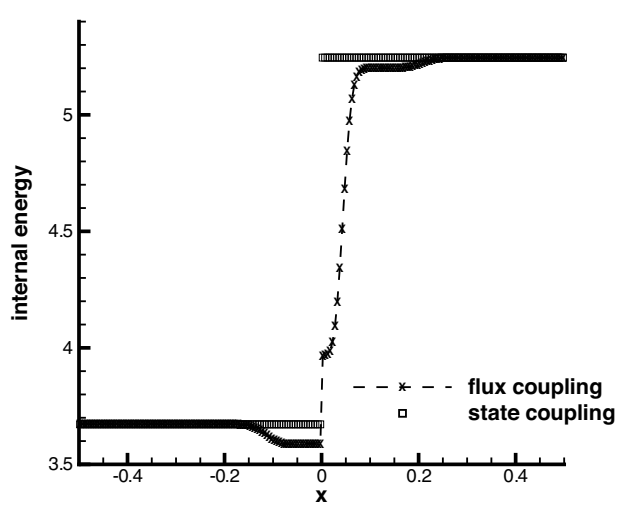

(d)

Figure 3. Conservative coupling vs. state coupling for test case 1: profiles of primitive variables $(\rho, u, p)$ and internal energy.

Results show that the uniform profile is not respected for the flux coupling. Nevertheless, the method is strictly conservative with zero conservation losses. In contrast, the uniform profiles of $\rho, u$ and $p$ are preserved when using state coupling as expected. Note also that the total energy is not conserved [10, 34. Finally, 


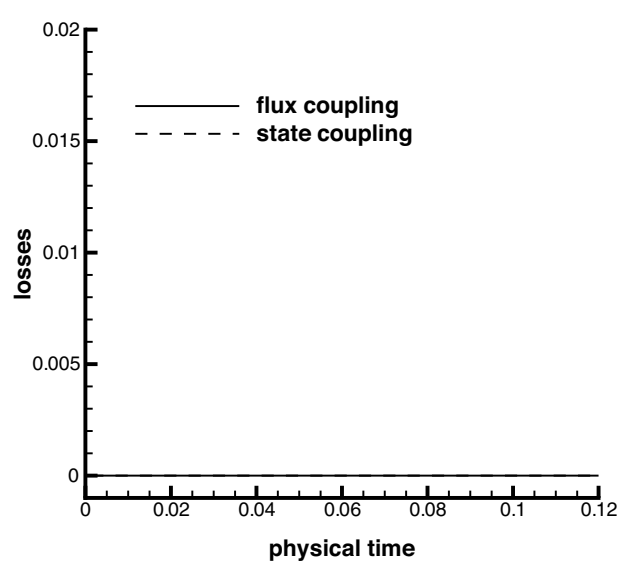

(a) relative mass conservation losses

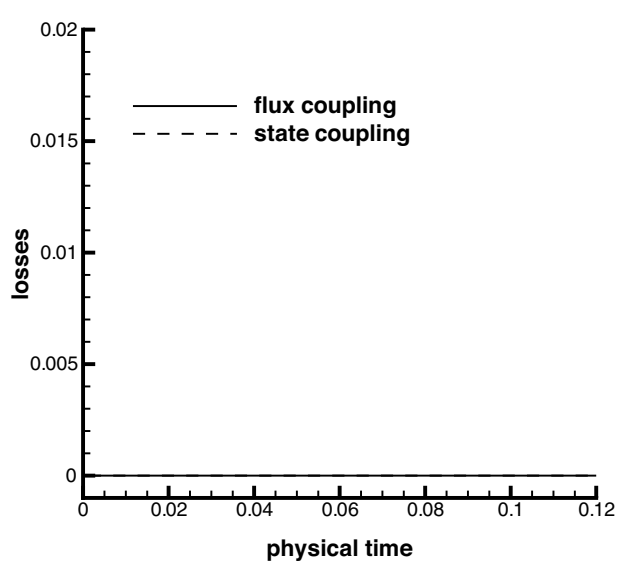

(b) relative momentum conservation losses

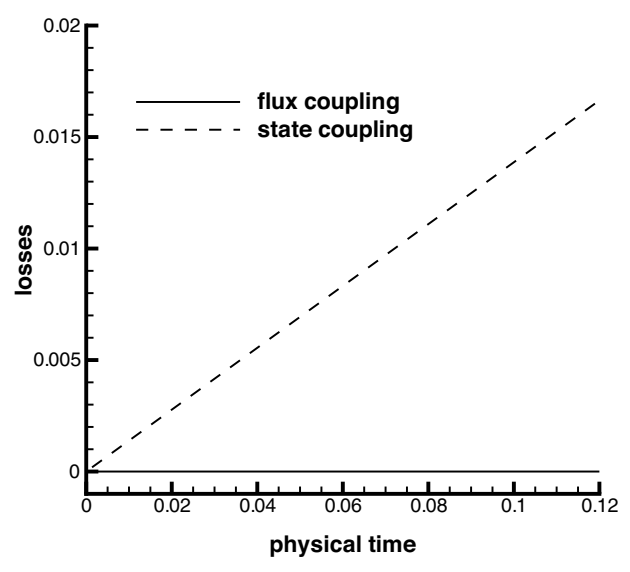

(c) relative energy conservation losses

FiguRE 4. Relative conservation losses for test case 1.

we remark that the internal energy $\varepsilon$ is necessarily discontinuous at the coupling interface due to the different pressure laws (see equation (2.2) ).

Test case 2. In the second test case, we still compare the various profiles based on conservative coupling or state coupling in primitive variables for a shock tube problem (see Table 1). The density, velocity, pressure, internal energy and mass flow rates are shown in Figure 5, while Figure 6 displays the relative conservation losses.

Results are similar to test case 1: the flux coupling method is strictly conservative and the state coupling method ensures continuity of primitive variables through the coupling interface.

4.2. Optimal source terms. Hereafter, $m_{r e f} \in \mathbb{R}^{*}$ and $\varepsilon_{r e f}>0$ will denote target values. At each time $t^{(n)}, n \in \mathbb{N}$, we consider the discrete states $\mathbf{U}_{L}=\mathbf{U}_{-\frac{1}{2}}^{(n)}$, and $\mathbf{U}_{R}=\mathbf{U}_{+\frac{1}{2}}^{(n)}$ at the coupling interface to define objective functions. 
For the numerical resolution of constrained optimization problems (3.38), we use the gradient projection algorithm defined by the sequence

$$
\left\{\begin{array}{l}
\widetilde{\mathcal{M}}_{0}^{e} \quad \text { given } \\
\widetilde{\mathcal{M}}_{k+1}^{e}=\mathcal{P}_{\mathcal{D}}\left(\widetilde{\mathcal{M}}_{k}^{e}-\mu_{\mathcal{J}} \nabla \mathcal{J}\left(\widetilde{\mathcal{M}}_{k}^{e}\right)\right), \quad k \in \mathbb{N}
\end{array}\right.
$$

where $\mathcal{P}_{\mathcal{D}}$ is the projection operator onto the set of constraints $\mathcal{D}$. In our numerical examples, $\mathcal{P}_{\mathcal{D}}$ is known explicitly and thus motivates the use of this algorithm. For a given tolerance $\eta>0$, the stopping condition of the algorithm will be $\| \widetilde{\mathcal{M}}_{k+1}^{e}-$ $\widetilde{\mathcal{M}}_{k}^{e}\|<\eta\| \widetilde{\mathcal{M}}_{0}^{e} \|$, where $\|\cdot\|$ denotes the usual Euclidean norm and $\eta=10^{-12}$.

Test case 3 . In this test case, we focus on an optimization problem that imposes the mass flow on the right-hand side of the coupling interface, while controlling the conservation losses in mass and momentum. The applications we have in mind here concern unsteady flow control techniques. Among the most widely used techniques, one can quote the experimental methods of fluid blowing or suction which have a strong influence on the flow features such as lift improvement, drag reduction, vortex breakdown delay, flow separation control [32, 33, 57]. In the experimental studies, a typical objective may be to optimize the blowing position for maximizing control and minimizing the blowing mass flow rate. A simplified representation of these flow control problems would consider the minimization of the objective function $\left(\mathcal{M}_{\rho}, \mathcal{M}_{\rho u}\right) \in \mathbb{R}^{2} \mapsto \mathcal{J}_{1}\left(\mathcal{M}_{\rho}, \mathcal{M}_{\rho u}\right) \in \mathbb{R}$ defined by

$$
\begin{aligned}
\mathcal{J}_{1}\left(\mathcal{M}_{\rho}, \mathcal{M}_{\rho u}\right):= & \left(\frac{m_{+}^{e}\left(\mathbf{u}_{L}, \mathbf{u}_{R}, \mathcal{M}_{\rho}, \mathcal{M}_{\rho u}\right)-m_{\text {ref }}}{m_{\text {ref }}}\right)^{2} \\
& +\kappa^{2}\left[\left(\frac{\mathcal{M}_{\rho u}}{a^{2} \tau_{R}}\right)^{2}+\left(\frac{\mathcal{M}_{\rho}}{a}\right)^{2}\right]
\end{aligned}
$$

over the set $\mathcal{D}_{1}=[-0.5,0.5]^{2}, \mathcal{J}_{1}$ is strictly convex (see the Appendix A). We further assume $\mathcal{M}_{\rho E}^{(n)}=0$, for all $n$ in $\mathbb{N}$. The parameter $\kappa>0$ quantifies the cost of the control. Minimizing $\mathcal{J}_{1}$ over $\mathbb{R}^{2}$ with $\kappa=0$ imposes $m_{+}^{e}=m_{\text {ref }}$, while conservation losses $\mathcal{M}_{\rho}$ and $\mathcal{M}_{\rho u}$ are set to zero when $\kappa$ tends to infinity. In this example we take $m_{\text {ref }}=0.75$.

Figure 7 represents the density, velocity, pressure, internal energy and mass flow profiles for three values of the control parameter: $\kappa=0.01, \kappa=1$ and $\kappa=$ 100 with respective parameter value $\mu_{\mathcal{J}}=0.1,10^{-2}$ and $10^{-4}$ in algorithm (4.1). These parameters were chosen experimentally to obtain a good convergence of the algorithm. Figure 8 displays the relative conservation losses in mass, momentum and total energy.

The total energy is conserved for all values of $\kappa$ as expected. Comparing Figures [5] and 7, we observe that the profiles of density, velocity, pressure, internal energy and mass flow obtained from the minimization of the cost function $\mathcal{J}_{1}$ with $\kappa=100$ are similar to those obtained by the flux coupling method. In contrast, the right trace of the mass flow tends to the value imposed $m_{\text {ref }}=0.75$ when $\kappa$ tends to zero. Finally, we observe that the amplitudes of the conservation losses in mass and momentum increase when the control parameter $\kappa$ decreases. 


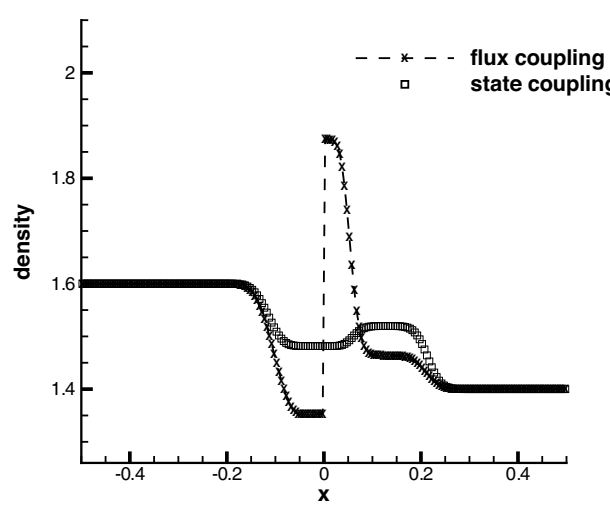

(a)

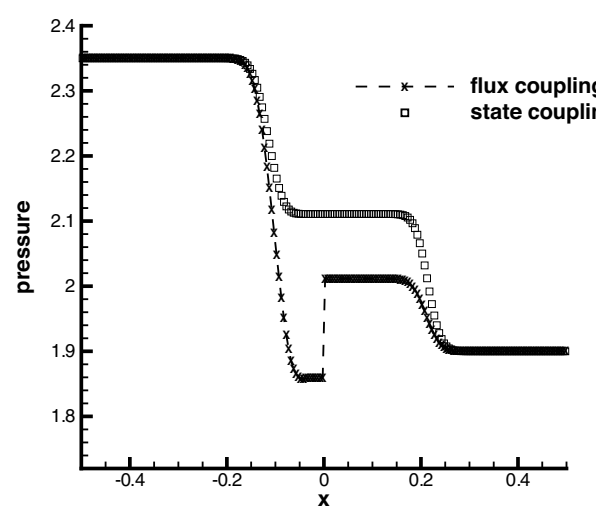

(c)

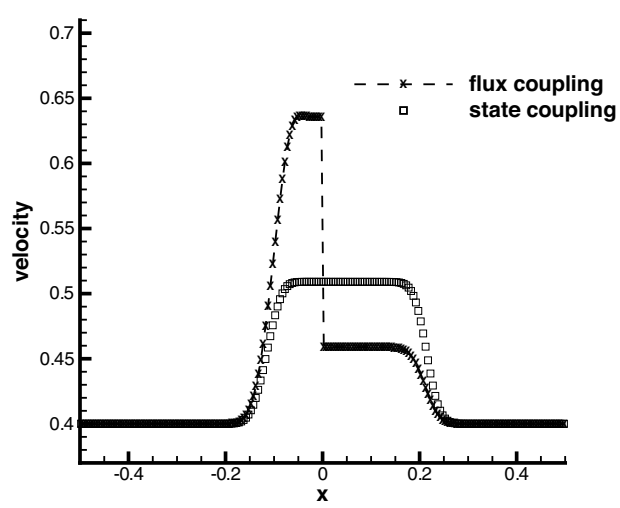

(b)

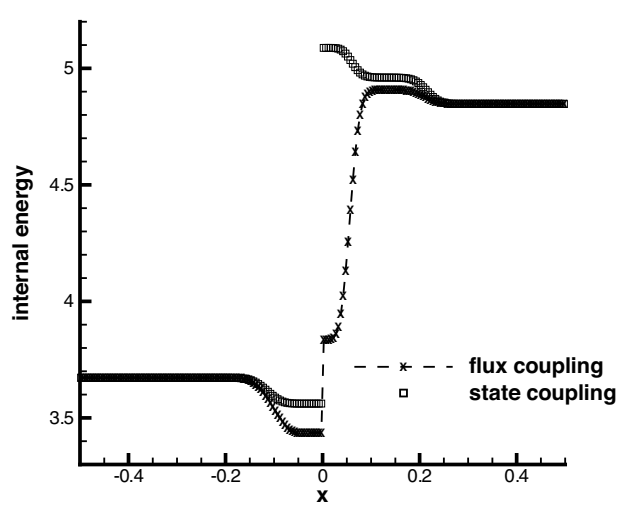

(d)

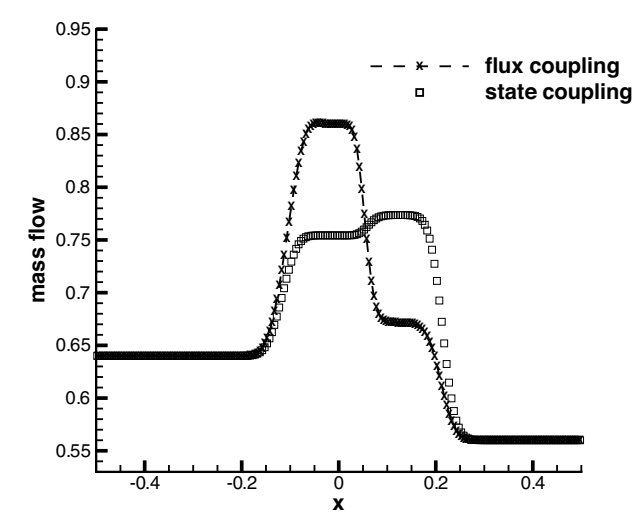

(e)

FIGURE 5. Conservative coupling vs. state coupling for test case 2: profiles of primitive variables $(\rho, u, p)$, mass flow and internal energy. 


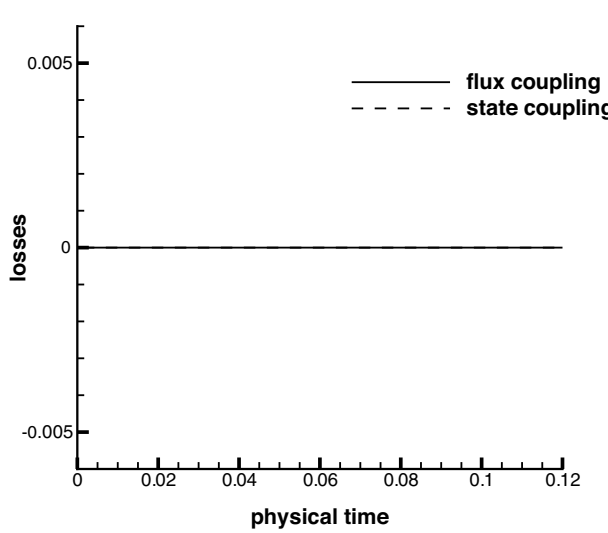

(a) relative mass conservation losses

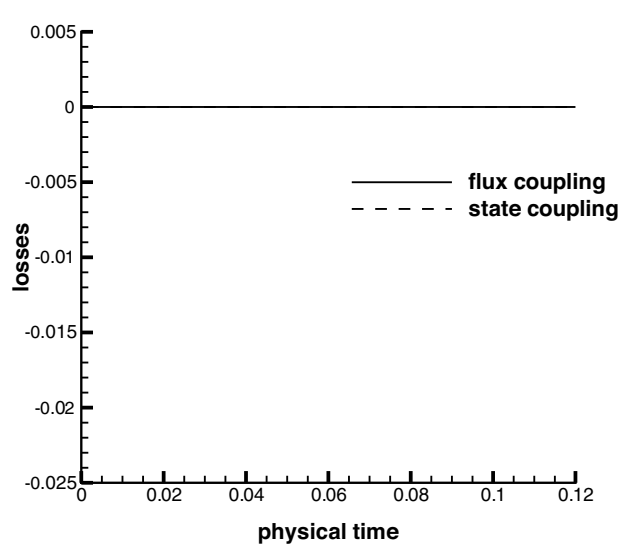

(b) relative momentum conservation losses

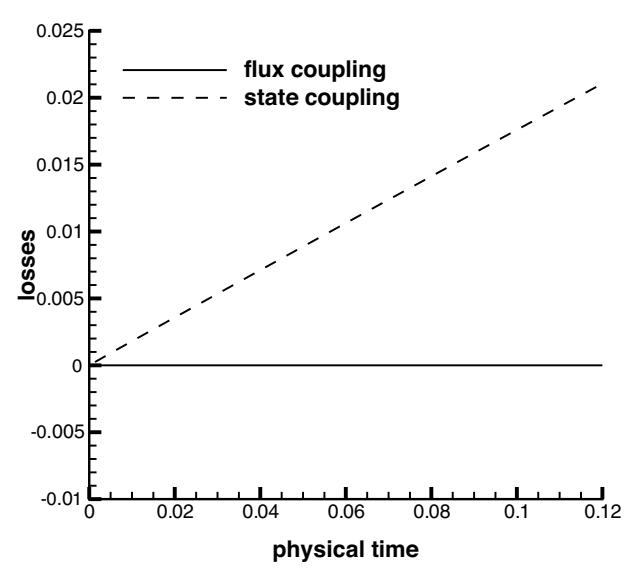

(c) relative energy conservation losses

Figure 6. Relative conservation losses for test case 2.

Test case 4. Now, for this test case, we set $\mathcal{M}_{\rho}^{(n)}=\mathcal{M}_{\rho u}^{(n)}=0$ at each time step and try to minimize the function $\mathcal{M}_{\rho E} \in \mathbb{R} \mapsto \mathcal{J}_{2}\left(\mathcal{M}_{\rho E}\right) \in \mathbb{R}$ defined by

$$
\mathcal{J}_{2}\left(\mathcal{M}_{\rho E}\right):=\left(\frac{\varepsilon_{+}-\varepsilon_{r e f}}{\varepsilon_{r e f}}\right)^{2}+\kappa^{2}\left(\frac{\mathcal{M}_{\rho E}}{a^{3} \tau_{R}^{2}}\right)^{2},
$$

with $\varepsilon_{\text {ref }}=3.5$ over the set $\mathcal{D}_{2}=[-1.5,1.5]$. This function is a strictly convex function of its argument (see Appendix A). It allows us to impose the temperature through the value of $\varepsilon_{+}$and thus imposes cooling $\left(\mathcal{M}_{\rho E}<0\right)$ or heating $\left(\mathcal{M}_{\rho E}>0\right)$ at the right of the interface $x=0$, while controlling the conservative losses. Film cooling constitutes an example of such a minimization problem. The efficiency of gas turbines may be improved and fuel consumption reduced with higher combustor exit flow temperatures. However, higher temperatures may induce turbine component damages. To insure their thermal protection, a protective coat of cool air separates the turbine wall from the hot gases 31. The boundary between the film and the gases can be modelled as an interface through which the intake air mass and energy losses must be considered. In Figure 9, we present the density, velocity, 


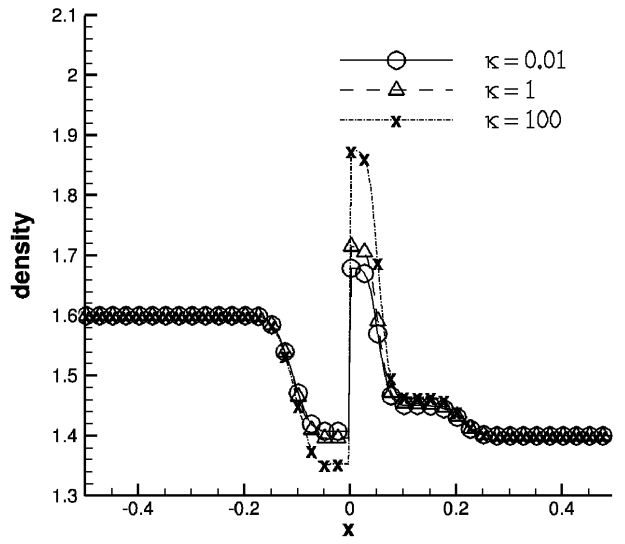

(a)

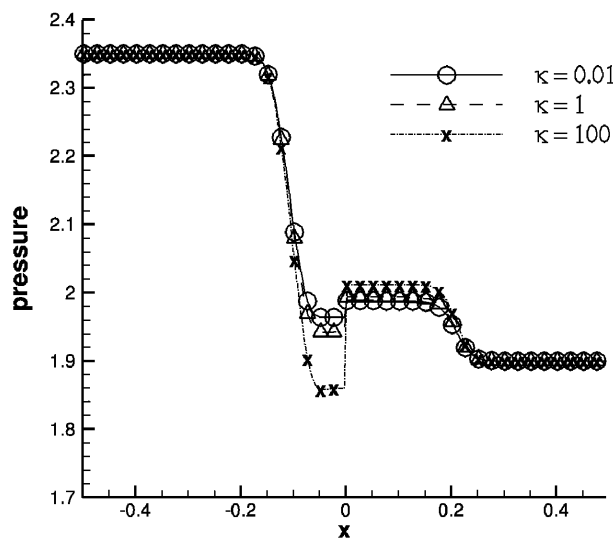

(c)

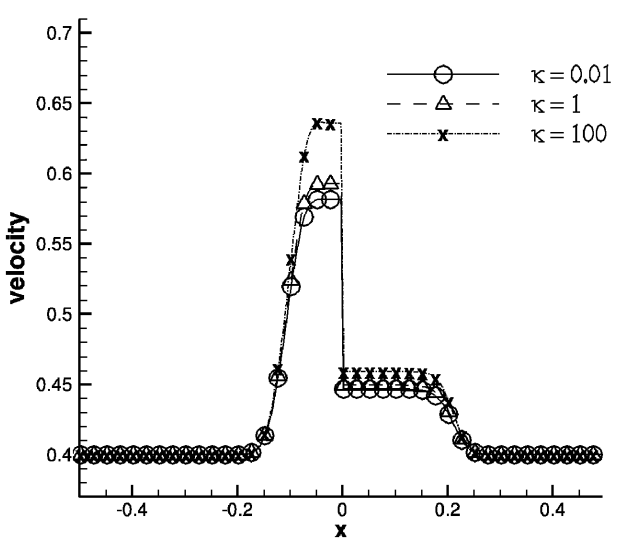

(b)

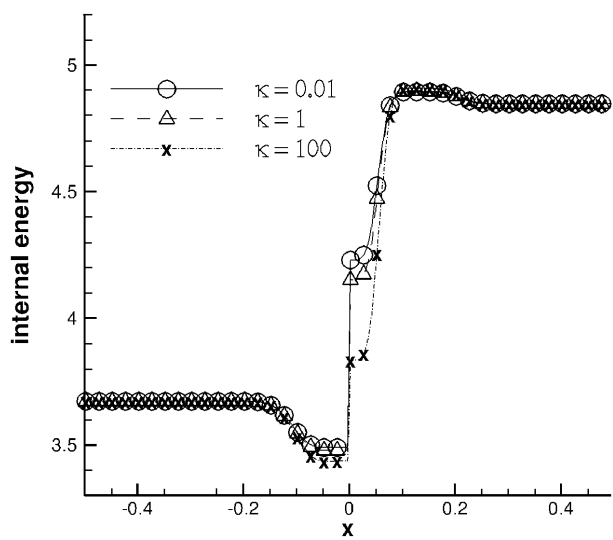

(d)

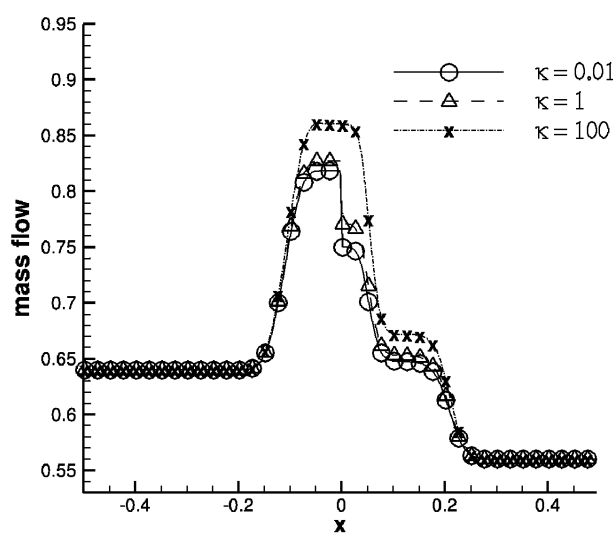

(e)

Figure 7. Constrained optimization for test case 3: profiles of primitive variables $(\rho, u, p)$, mass flow and internal energy. 


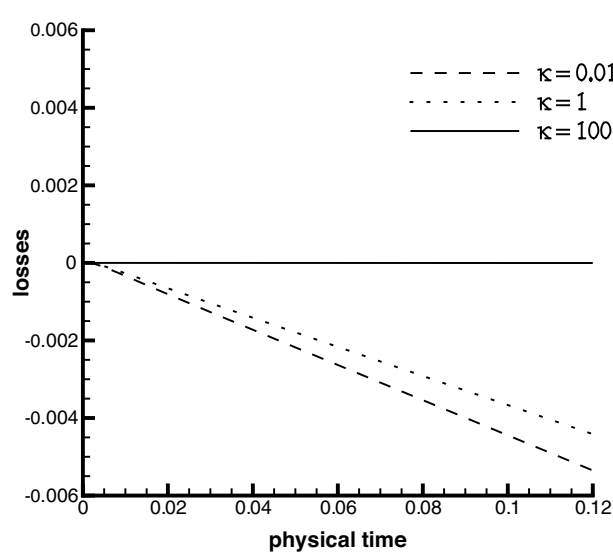

(a) relative mass conservation losses

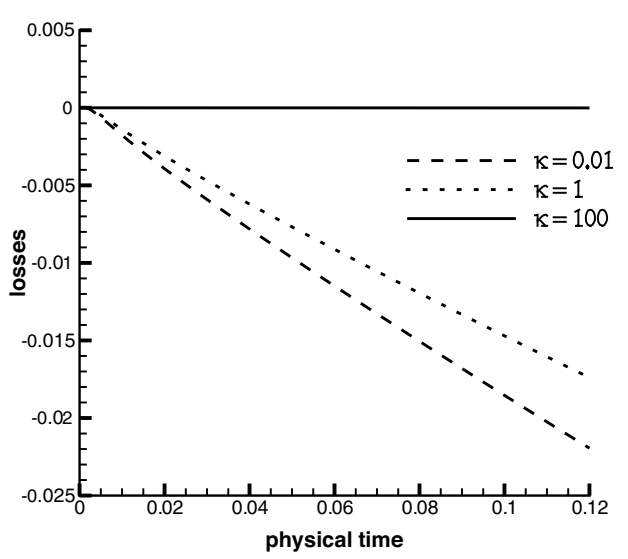

(b) relative momentum conservation losses

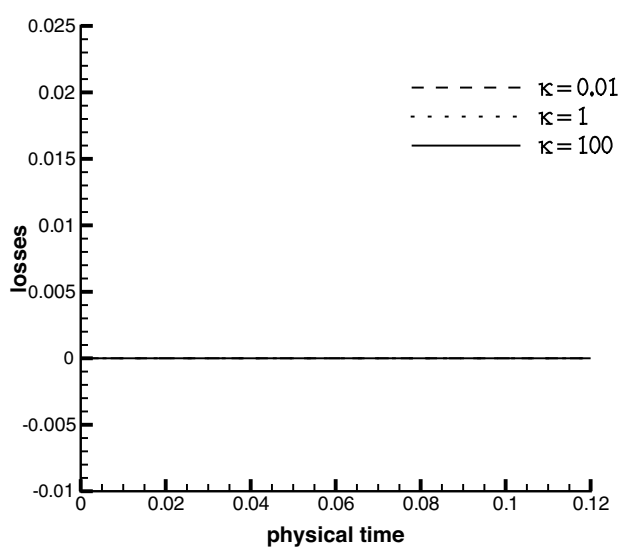

(c) relative energy conservation losses

FiguRE 8. Relative conservation losses for test case 3.

pressure, internal energy and mass flow profiles for different values of the control parameter. Figure 10 displays the relative conservation losses for these values of $\kappa$. Here again, the conclusions are similar to those of the previous test case.

Test case 5. Finally, in this last test case, we assume that $\mathcal{M}_{\rho u}^{(n)}=0$ for all $n$ in $\mathbb{N}$ and minimize the function $\left(\mathcal{M}_{\rho}, \mathcal{M}_{\rho E}\right) \in \mathbb{R}^{2} \mapsto \mathcal{J}_{3}\left(\mathcal{M}_{\rho}, \mathcal{M}_{\rho E}\right) \in \mathbb{R}$ defined by

$$
\mathcal{J}_{3}\left(\mathcal{M}_{\rho}, \mathcal{M}_{\rho E}\right):=\left(\frac{\varepsilon_{+}-\varepsilon_{r e f}}{\varepsilon_{r e f}}\right)^{2}+\kappa^{2}\left[\left(\frac{\mathcal{M}_{\rho}}{a}\right)^{2}+\left(\frac{\mathcal{M}_{\rho E}}{a^{3} \tau_{R}^{2}}\right)^{2}\right],
$$

over the set $\mathcal{D}_{3}=[0,0.5] \times[-0.5,0]$. We set $\varepsilon_{r e f}=3.5$, this function imposes the temperature while controlling the conservative losses. The main difference consists in film cooling by injection of mass $\left(\mathcal{M}_{\rho}>0\right)$ and loss of energy $\left(\mathcal{M}_{\rho E}<0\right)$. Figure 11 presents the density, velocity, pressure, internal energy and mass flow profiles as a function of the control parameter. Figure 12 displays the relative conservation losses for these three values of $\kappa$. Here again, the conclusions are similar to the other optimization problems. 


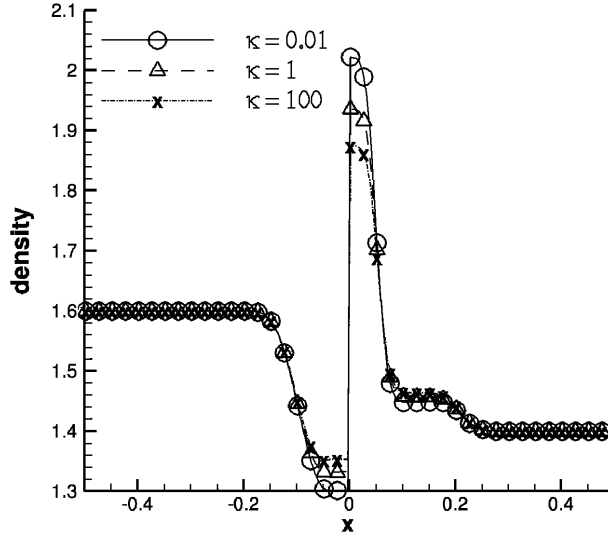

(a)

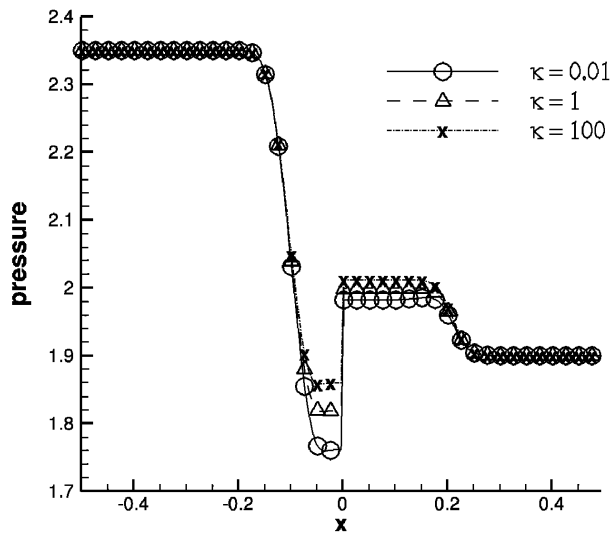

(c)

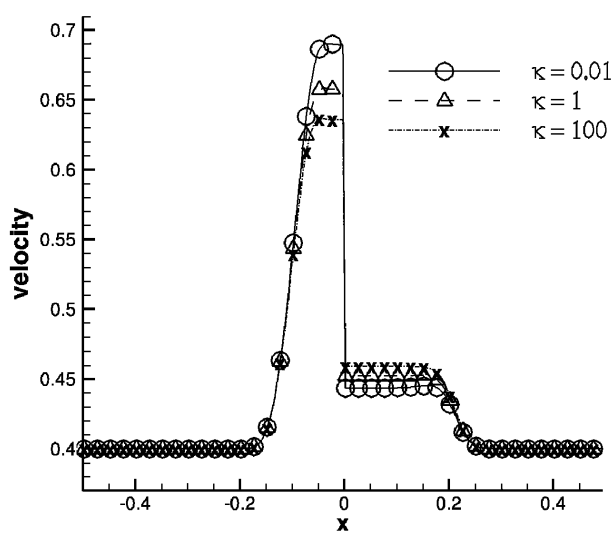

(b)

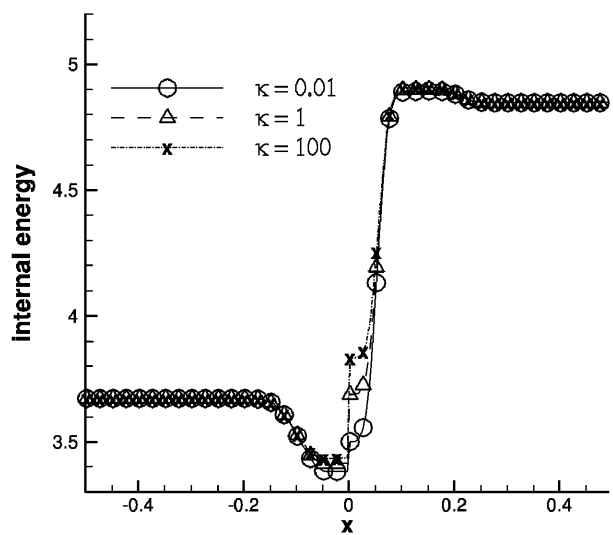

(d)

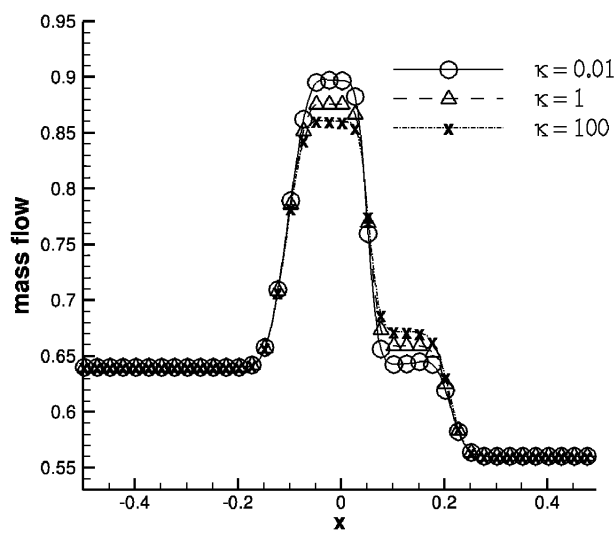

(e)

Figure 9. Constrained optimization for test case 4: profiles of primitive variables $(\rho, u, p)$, mass flow and internal energy. 


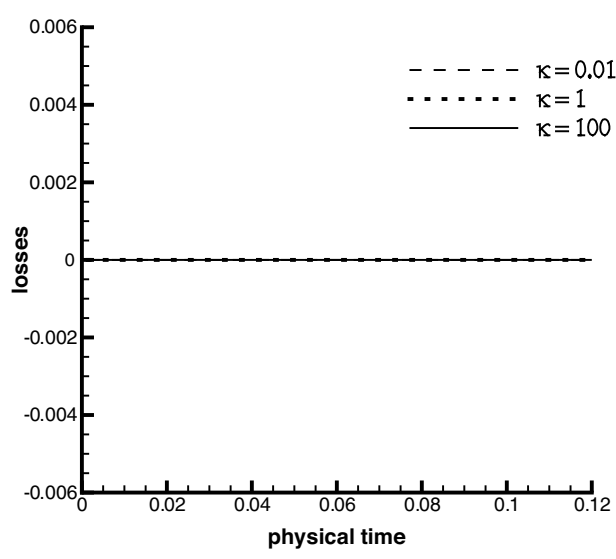

(a) relative mass conservation losses

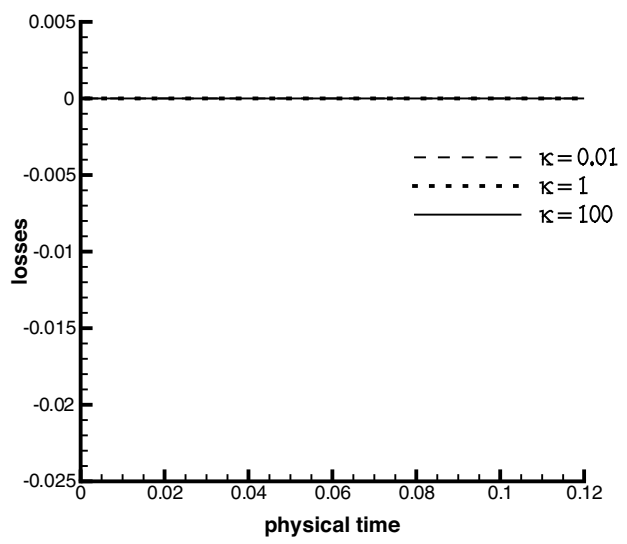

(b) relative momentum conservation losses

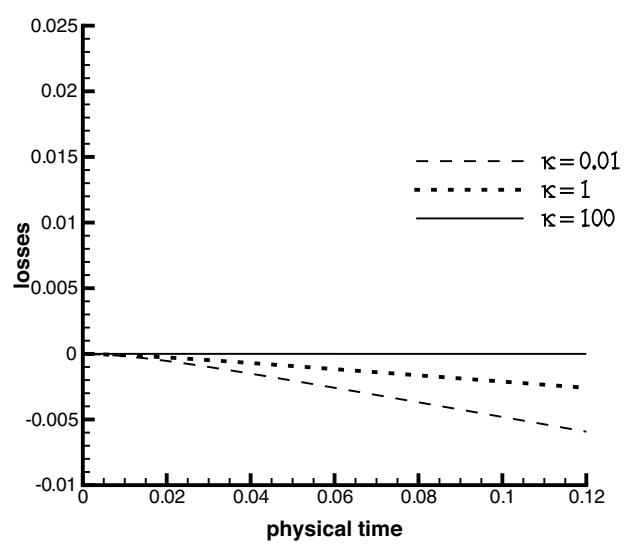

(c) relative energy conservation losses

Figure 10. Relative conservation losses for test case 4. 


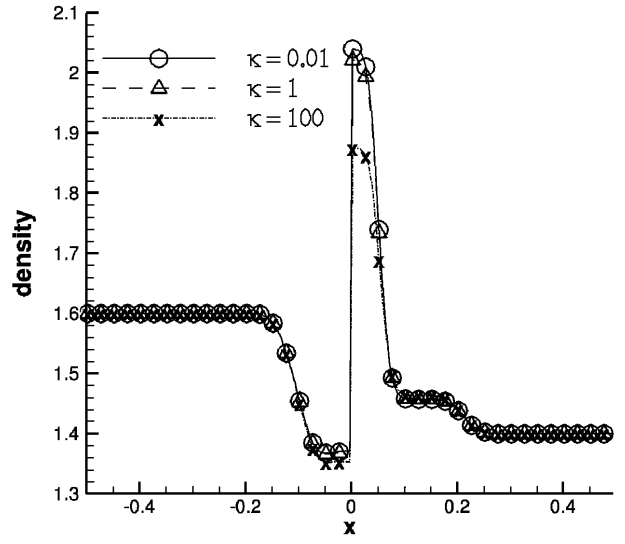

(a)

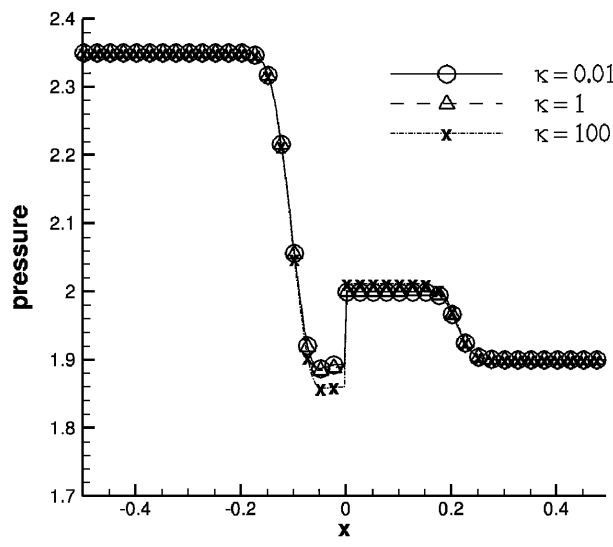

(c)

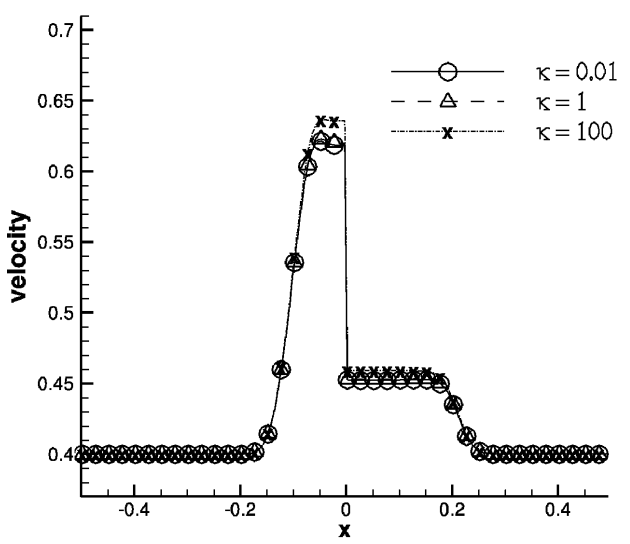

(b)

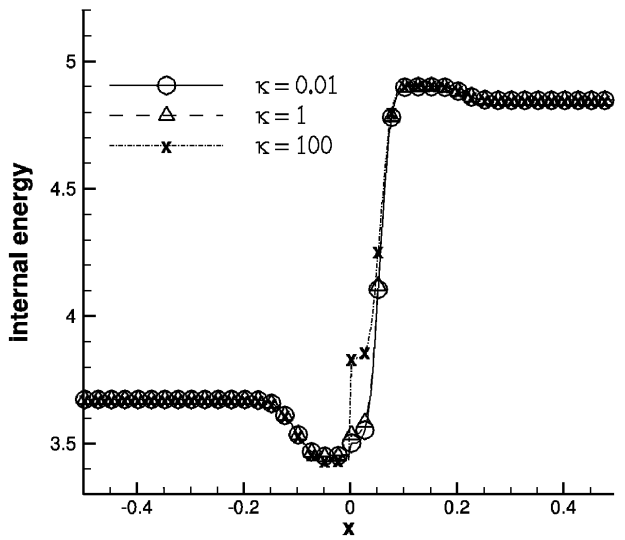

(d)

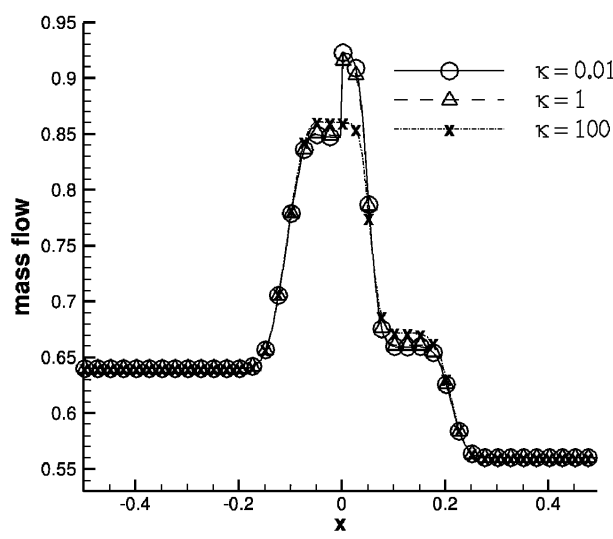

(e)

Figure 11. Constrained optimization for test case 5: profiles of primitive variables $(\rho, u, p)$, mass flow and internal energy. 


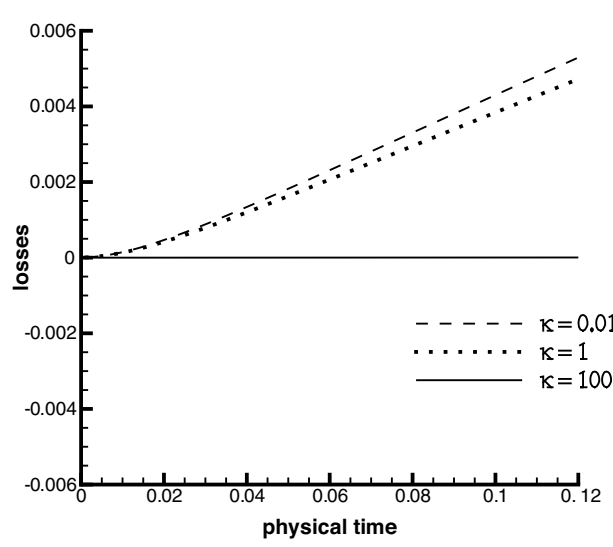

(a) relative mass conservation losses

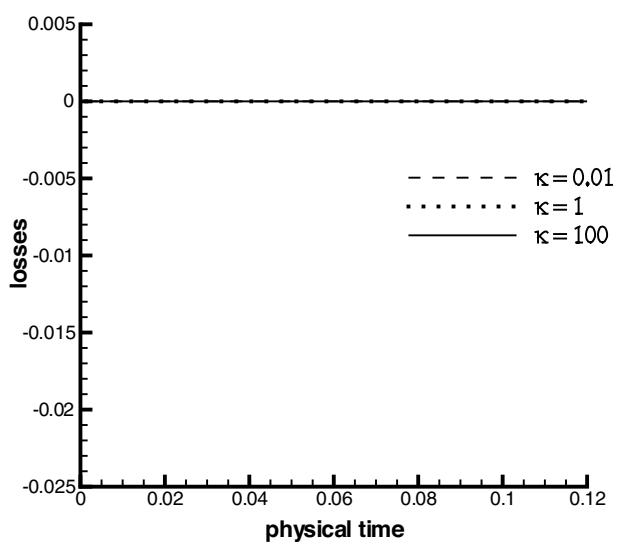

(b) relative momentum conservation losses

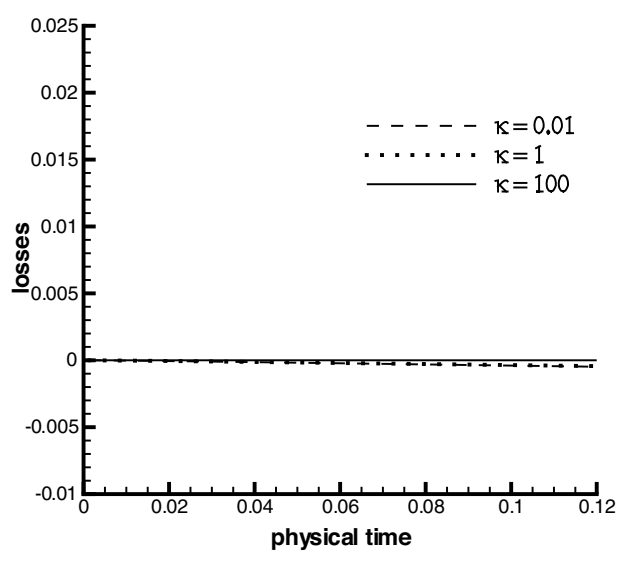

(c) relative energy conservation losses

FiguRE 12. Relative conservation losses for test case 5.

\section{Concluding Remarks}

We have extended the study of interface coupling with local measure source term [4, 5, 34] to the general case where all the source terms are nonzero. An approximate Riemann solver preserving equilibrium solutions has been proposed for the numerical approximation of the coupled model in the context of the gas dynamics equations with different closure laws for the pressure, as a model example. The construction of this solver relies on a relaxation approximation where the associated Riemann problem has been solved analytically. This relaxation method has been used for the evaluation of optimal source terms satisfying physical criteria at the coupling interface. In the context of constrained optimization problems, numerical experiments have shown that it is possible to impose physical quantities such as mass flow or temperature at the coupling interface while controlling conservation losses. Future investigations should include an extension to several space dimensions and adaptive procedures, together with the coupling of more realistic models 
(even if we cannot expect explicit theoretical results for general models, at least it is interesting to extend the numerical approach to less academic cases).

\section{Appendix A. Mathematical properties of the cost functions $\mathcal{J}_{1}$ AND $\mathcal{J}_{2}$}

First, recall that the sequence of the gradient projection algorithm converges to the optimum if the objective function $\mathcal{J}$ is $\alpha_{\mathcal{J}}$-convex differentiable and $\nabla \mathcal{J}$ is $\mathcal{C}_{\nabla \mathcal{J}}$-Lipschitz continuous. More precisely, if the algorithm parameter $\mu_{\mathcal{J}}$ is chosen such that the inequality

holds, then the algorithm converges.

$$
0<\mu_{\mathcal{J}}<\frac{2 \alpha_{\mathcal{J}}}{\mathcal{C}_{\nabla \mathcal{J}}^{2}},
$$

Here, we give some properties of the cost functions $\mathcal{J}_{1}$ and $\mathcal{J}_{2}$ respectively defined in (4.2) and (4.3).

Proposition A.1. For all control parameters $\kappa>0$, the functions $\mathcal{J}_{1}$ and $\mathcal{J}_{2}$ are strictly convex. For each $\kappa>0$, set

$$
\begin{gathered}
\alpha_{1}=\frac{1}{2}\left[\operatorname{Tr}\left(\operatorname{Hess}\left(\mathcal{J}_{1}\right)\right)-\sqrt{\operatorname{Tr}\left(\operatorname{Hess}\left(\mathcal{J}_{1}\right)\right)^{2}-4 \operatorname{det}\left(\operatorname{Hess}\left(\mathcal{J}_{1}\right)\right)}\right]>0, \\
\mathcal{C}_{\nabla \mathcal{J}_{1}}=\operatorname{rspec}\left(\operatorname{Hess}\left(\mathcal{J}_{1}\right)\right)>0, \\
\alpha_{2}=\frac{2 \kappa^{2}}{a^{6} \tau_{R}^{4}}>0, \quad \mathcal{C}_{\nabla \mathcal{J}_{2}}=\frac{2}{\left(\varepsilon_{\text {ref }} m_{+}\right)^{2}}+\alpha_{2}>0,
\end{gathered}
$$

where for a matrix $A, \operatorname{Tr}(A)$ denotes its trace, $\operatorname{det}(A)$ its determinant and $\operatorname{rspec}(A)$ its spectral radius. Hess $\left(\mathcal{J}_{1}\right)$ denotes the Hessian matrix of the cost function $\mathcal{J}_{1}$. Then, for $i=1,2$, there exists $\alpha_{\mathcal{J}_{i}}$ strictly positive and strictly smaller than $\alpha_{i}$ such that the function $\mathcal{J}_{i}$ is $\alpha_{\mathcal{J}_{i}}$-convex differentiable and $\nabla \mathcal{J}_{i}$ is $\mathcal{C}_{\nabla \mathcal{J}_{i}}$-Lipschitz. Finally, for $i=1,2$, the gradient projection algorithm converges for all parameters $\mu_{\mathcal{J}_{i}}$ verifying:

$$
0<\mu_{\mathcal{J}_{i}}<\frac{2 \alpha_{\mathcal{J}_{i}}}{\mathcal{C}_{\nabla \mathcal{J}_{i}}^{2}}
$$

Proof. First of all, one can check that $\operatorname{Tr}\left(\operatorname{Hess}\left(\mathcal{J}_{1}\right)\right)>0$ and $\operatorname{det}\left(\operatorname{Hess}\left(\mathcal{J}_{1}\right)\right)>0$ for all $\kappa>0$, since after easy calculations we get

and

$$
\operatorname{Tr}\left(\operatorname{Hess}\left(\mathcal{J}_{1}\right)\right)=\frac{2\left(a \tau_{L}^{*}-u^{*}\right)^{2}}{\left[a m_{r e f}\left(\tau_{L}^{*}+\tau_{R}^{*}\right)\right]^{2}}+\frac{2 \kappa^{2}}{a^{2}}+\frac{2}{\left[a m_{r e f}\left(\tau_{L}^{*}+\tau_{R}^{*}\right)\right]^{2}}+\frac{2 \kappa^{2}}{a^{4} \tau_{R}^{2}}>0
$$

$$
\operatorname{det}\left(\operatorname{Hess}\left(\mathcal{J}_{1}\right)\right)=\frac{4 \kappa^{2}}{a^{4}}\left[\frac{\left(a \tau_{L}^{*}-u^{*}\right)^{2}}{\left[a m_{r e f} \tau_{R}\left(\tau_{L}^{*}+\tau_{R}^{*}\right)\right]^{2}}+\frac{1}{\left[m_{r e f}\left(\tau_{L}^{*}+\tau_{R}^{*}\right)\right]^{2}}+\frac{\kappa^{2}}{a^{2} \tau_{R}^{2}}\right]>0 .
$$

This implies, in particular, the strict convexity of $\mathcal{J}_{1}$ since its Hessian matrix is positive definite. Let

$$
\Delta=\operatorname{Tr}\left(\operatorname{Hess}\left(\mathcal{J}_{1}\right)\right)^{2}-4 \operatorname{det}\left(\operatorname{Hess}\left(\mathcal{J}_{1}\right)\right) .
$$

First, note that

$$
\begin{aligned}
\Delta & =\left[\partial_{\mathcal{M}_{\rho}^{2}}^{2} \mathcal{J}_{1}+\partial_{\mathcal{M}_{\rho u}^{2}}^{2} \mathcal{J}_{1}\right]^{2}-4\left[\partial_{\mathcal{M}_{\rho}^{2}}^{2} \mathcal{J}_{1} \partial_{\mathcal{M}_{\rho u}^{2}}^{2} \mathcal{J}_{1}-\left(\partial_{\mathcal{M}_{\rho}, \mathcal{M}_{\rho u}}^{2} \mathcal{J}_{1}\right)^{2}\right] \\
& =\left[\partial_{\mathcal{M}_{\rho}^{2}}^{2} \mathcal{J}_{1}-\partial_{\mathcal{M}_{\rho u}^{2}}^{2} \mathcal{J}_{1}\right]^{2}+4\left(\partial_{\mathcal{M}_{\rho}, \mathcal{M}_{\rho u}}^{2} \mathcal{J}_{1}\right)^{2}>0,
\end{aligned}
$$


since

$$
\partial_{\mathcal{M}_{\rho}, \mathcal{M}_{\rho u}}^{2} \mathcal{J}_{1}=\frac{2\left(a \tau_{L}^{*}-u^{*}\right)}{\left[a m_{r e f}\left(\tau_{L}^{*}+\tau_{R}^{*}\right)\right]^{2}} \neq 0 \quad\left(\text { because } \lambda_{1}^{r}<0\right),
$$

and then observe that

$$
\Delta-\operatorname{Tr}\left(\operatorname{Hess}\left(\mathcal{J}_{1}\right)\right)^{2}=-4 \operatorname{det}\left(\operatorname{Hess}\left(\mathcal{J}_{1}\right)\right)<0 .
$$

From $\Delta>0, \operatorname{Tr}\left(\operatorname{Hess}\left(\mathcal{J}_{1}\right)\right)>0$ and the previous inequality, we deduce that the term $\alpha_{1}$ defined in the present proposition is strictly positive. So, there exists $\alpha_{\mathcal{J}_{1}}$ such that $0<\alpha_{\mathcal{J}_{1}}<\alpha_{1}$. Now, in order to show that $\mathcal{J}_{1}$ is $\alpha_{\mathcal{J}_{1}}$-convex, let us define the function

$$
f_{1}: \quad\left(\mathcal{M}_{\rho}, \mathcal{M}_{\rho u}\right) \in \mathbb{R}^{2} \longmapsto \mathcal{J}_{1}\left(\mathcal{M}_{\rho}, \mathcal{M}_{\rho u}\right)-\frac{\alpha_{\mathcal{J}_{1}}}{2}\left\|\left(\mathcal{M}_{\rho}, \mathcal{M}_{\rho u}\right)\right\|^{2} \in \mathbb{R}
$$

where $\|\cdot\|$ denotes the usual Euclidean norm on $\mathbb{R}^{2}$. After easy calculations, we obtain

$$
\operatorname{Tr}\left(\operatorname{Hess}\left(f_{1}\right)\right)=\operatorname{Tr}\left(\operatorname{Hess}\left(\mathcal{J}_{1}\right)\right)-2 \alpha_{\mathcal{J}_{1}}
$$

and

$$
\operatorname{det}\left(\operatorname{Hess}\left(f_{1}\right)\right)=\operatorname{det}\left(\operatorname{Hess}\left(\mathcal{J}_{1}\right)\right)-\alpha_{\mathcal{J}_{1}} \operatorname{Tr}\left(\operatorname{Hess}\left(\mathcal{J}_{1}\right)\right)+\alpha_{\mathcal{J}_{1}}^{2}
$$

Since $\alpha_{\mathcal{J}_{1}}<\alpha_{1}<\operatorname{Tr}\left(\operatorname{Hess}\left(\mathcal{J}_{1}\right)\right) / 2$, it is clear that the trace of the Hessian matrix of the function $f_{1}$ is strictly positive. Moreover, considering the polynomial of degree 2 in $X$,

$$
\mathcal{P}(X)=X^{2}-\operatorname{Tr}\left(\operatorname{Hess}\left(\mathcal{J}_{1}\right)\right) X+\operatorname{det}\left(\operatorname{Hess}\left(\mathcal{J}_{1}\right)\right)
$$

one can see that its discriminant is given by $\Delta>0$ in (1.1) and $\alpha_{1}$ is its smallest root. Since $\alpha_{\mathcal{J}_{1}}<\alpha_{1}$, the determinant of the Hessian matrix of the function $f_{1}$ is strictly positive and we deduce that $f_{1}$ is in particular convex, that is to say, that $\mathcal{J}_{1}$ is $\alpha_{\mathcal{J}_{1}}$ convex. For all $\kappa>0$, the gradient $\nabla \mathcal{J}_{1}$ of the function $\mathcal{J}_{1}$ is Lipschitz-continuous with constant $\mathcal{C}_{\nabla \mathcal{J}_{1}}$ since the eigenvalues of the Hessian of $\mathcal{J}_{1}$ are bounded above by $\mathcal{C}_{\nabla \mathcal{J}_{1}}$. The constant $\mathcal{C}_{\nabla \mathcal{J}_{1}}$ is strictly positive because $\mathcal{J}_{1}$ is strictly convex and is independent of the variables $\mathcal{M}_{\rho}$ and $\mathcal{M}_{\rho u}$ because the Hessian is independent of these variables.

Now, let us introduce $0<\alpha_{\mathcal{J}_{2}}<\alpha_{2}$ and the function

$$
f_{2}: \quad \mathcal{M}_{\rho E} \in \mathbb{R} \longmapsto \mathcal{J}_{2}\left(\mathcal{M}_{\rho E}\right)-\frac{\alpha_{\mathcal{J}_{2}}}{2} \mathcal{M}_{\rho E}^{2} \in \mathbb{R}
$$

Then, the second order derivative of $\mathcal{J}_{2}$ is given by

$$
\mathcal{J}_{2}^{\prime \prime}\left(\mathcal{M}_{\rho E}\right)=\left\{\begin{array}{llc}
\frac{2 \kappa^{2}}{a^{6} \tau_{R}^{4}} & \text { if } \quad \mathcal{M}_{\rho E} \in \mathcal{D}_{a d m}^{-}, \\
\frac{2 \kappa^{2}}{a^{6} \tau_{R}^{4}}+\frac{2}{\varepsilon_{r e f}^{2} m_{+}^{2}} & \text { if } & \mathcal{M}_{\rho E} \in \mathcal{D}_{a d m}^{+},
\end{array}\right.
$$

which implies $f_{2}^{\prime \prime}\left(\mathcal{M}_{\rho E}\right)=\mathcal{J}_{2}^{\prime \prime}\left(\mathcal{M}_{\rho E}\right)-\alpha_{\mathcal{J}_{2}}>0$; that is to say, that the function $\mathcal{J}_{2}$ is $\alpha_{\mathcal{J}_{2}}$-convex. Moreover, given the above relations, we directly obtain

$$
\sup \left|\mathcal{J}_{2}^{\prime \prime}\left(\mathcal{M}_{\rho E}\right)\right| \leq \mathcal{C}_{\nabla \mathcal{J}_{2}}
$$

and applying a corollary of the mean value theorem, we deduce that for all $\kappa>0$, the gradient $\nabla \mathcal{J}_{2}$ is Lipschitz-continuous with constant $\mathcal{C}_{\nabla \mathcal{J}_{2}}$. This concludes the proof. 


\section{ACKNOWLEDGEMENTS}

The authors warmly thank the reviewers for their constructive comments which helped them to improve the content of the first version of the manuscript. They were very interested to make connections with previous works which could shed light on some features of their modeling and discretizing approach which they had not yet perceived.

The second author acknowledges the support of the LRC Manon (Modélisation et approximation numérique orientées pour l'énergie nucléaire - CEA/DM2S-LJLL).

\section{REFERENCES}

[1] R. Abgrall and S. Karni, Computations of compressible multifluids, J. Comput. Phys. 169 (2001), no. 2, 594-623, DOI 10.1006/jcph.2000.6685. MR1836526 (2002b:76077)

[2] D. Amadori, L. Gosse, and G. Guerra, Godunov-type approximation for a general resonant balance law with large data, J. Differential Equations 198 (2004), no. 2, 233-274, DOI 10.1016/j.jde.2003.10.004. MR2038581 (2004m:65114)

[3] L. Ambrosio, A. Bressan, D. Helbing, A. Klar, and E. Zuazua, Modelling and optimisation of flows on networks, Lecture Notes in Mathematics, vol. 2062, Springer, Heidelberg; Fondazione C.I.M.E., Florence, 2013. MR3075424

[4] A. Ambroso, C. Chalons, F. Coquel, T. Galié, Interface model coupling via prescribed local flux balance, 18th AIAA Computational Fluid Dynamics Conference, AIAA paper 2007-3822, 2007.

[5] A. Ambroso, C. Chalons, F. Coquel, and T. Galié, Interface model coupling via prescribed local flux balance, ESAIM Math. Model. Numer. Anal. 48 (2014), no. 3, 895-918, DOI 10.1051/m2an/2013125. MR3264339

[6] A. Ambroso, C. Chalons, F. Coquel, T. Galié, E. Godlewski, F. Lagoutière, P-A. Raviart, N. Seguin, Numerical coupling of two-phase flows, Recent progress in scientific computing (SCPDE, Hong Kong), 168-178, 2005.

[7] A. Ambroso, C. Chalons, F. Coquel, E. Godlewski, F. Lagoutière, P.-A. Raviart, and N. Seguin, The coupling of homogeneous models for two-phase flows, Int. J. Finite Vol. 4 (2007), no. 1, 39. MR2465468 (2009m:76058)

[8] A. Ambroso, C. Chalons, F. Coquel, E. Godlewski, F. Lagoutière, P.-A. Raviart, and N. Seguin, Coupling of general Lagrangian systems, Math. Comp. 77 (2008), no. 262, 909-941, DOI 10.1090/S0025-5718-07-02064-9. MR2373185 (2008k:35308)

[9] A. Ambroso, C. Chalons, F. Coquel, E. Godlewski, F. Lagoutière, P.-A. Raviart, and N. Seguin, Relaxation methods and coupling procedures, Internat. J. Numer. Methods Fluids 56 (2008), no. 8, 1123-1129, DOI 10.1002/fld.1680. MR2393506

[10] A. Ambroso, C. Chalons, F. Coquel, E. Godlewski, F. Lagoutière, P-A. Raviart, N. Seguin, J-M. Hérard, Coupling of multiphase flow models, Proceedings of the Eleventh International Meeting on Nuclear Thermal-Hydraulics (NURETH), 2005.

[11] B. Andreianov, K. H. Karlsen, and N. H. Risebro, A theory of $L^{1}$-dissipative solvers for scalar conservation laws with discontinuous flux, Arch. Ration. Mech. Anal. 201 (2011), no. 1, 27-86, DOI 10.1007/s00205-010-0389-4. MR2807133

[12] B. Andreianov, F. Lagoutière, N. Seguin, and T. Takahashi, Small solids in an inviscid fluid, Netw. Heterog. Media 5 (2010), no. 3, 385-404, DOI 10.3934/nhm.2010.5.385. MR2670647 (2011k:76012)

[13] B. Andreianov and N. Seguin, Analysis of a Burgers equation with singular resonant source term and convergence of well-balanced schemes, Discrete Contin. Dyn. Syst. 32 (2012), no. 6, 1939-1964, DOI 10.3934/dcds.2012.32.1939. MR.2885792

[14] M. Arienti, P. Hung, E. Morano, J. E. Shepherd, A level set approach to Eulerian-Lagrangian coupling, J. Comput. Phys., 185, 213-251, 2003.

[15] F. Bouchut, Construction of BGK models with a family of kinetic entropies for a given system of conservation laws, J. Statist. Phys. 95 (1999), no. 1-2, 113-170, DOI 10.1023/A:1004525427365. MR:1705583(2000f:82084) 
[16] F. Bouchut, Nonlinear stability of finite volume methods for hyperbolic conservation laws and well-balanced schemes for sources, Frontiers in Mathematics, Birkhäuser Verlag, Basel, 2004. MR2128209(2005m:65002)

[17] F. Bouchut, A reduced stability condition for nonlinear relaxation to conservation laws, J. Hyperbolic Differ. Equ. 1 (2004), no. 1, 149-170, DOI 10.1142/S0219891604000020. MR2052474 (2005k:35258)

[18] B. Boutin, Étude mathématique et numérique d'équations hyperboliques non-linéaires: couplage de modèles et chocs non classiques, PhD thesis, Université Pierre et Marie Curie-Paris VI, France, 2009.

[19] C. Castro, F. Palacios, and E. Zuazua, An alternating descent method for the optimal control of the inviscid Burgers equation in the presence of shocks, Math. Models Methods Appl. Sci. 18 (2008), no. 3, 369-416, DOI 10.1142/S0218202508002723. MR2397976 (2009c:35392)

[20] C. Chalons and F. Coquel, Navier-Stokes equations with several independent pressure laws and explicit predictor-corrector schemes, Numer. Math. 101 (2005), no. 3, 451-478, DOI 10.1007/s00211-005-0612-7. MR.2194824 (2006m:76119)

[21] C. Chalons, F. Coquel, E. Godlewski, P.-A. Raviart, and N. Seguin, Godunov-type schemes for hyperbolic systems with parameter-dependent source. The case of Euler system with friction, Math. Models Methods Appl. Sci. 20 (2010), no. 11, 2109-2166, DOI 10.1142/S021820251000488X. MR2740716 (2011m:65179)

[22] C. Chalons and J.-F. Coulombel, Relaxation approximation of the Euler equations, J. Math. Anal. Appl. 348 (2008), no. 2, 872-893, DOI 10.1016/j.jmaa.2008.07.034. MR2446042 (2010b:35349)

[23] C. Chalons, P.-A. Raviart, and N. Seguin, The interface coupling of the gas dynamics equations, Quart. Appl. Math. 66 (2008), no. 4, 659-705, DOI 10.1090/S0033-569X-08-01087-X. MR.2465140(2009k:76113)

[24] G. Q. Chen, C. D. Levermore, and T.-P. Liu, Hyperbolic conservation laws with stiff relaxation terms and entropy, Comm. Pure Appl. Math. 47 (1994), no. 6, 787-830, DOI 10.1002/cpa.3160470602. MR1280989 (95h:35133)

[25] F. Coquel, E. Godlewski, B. Perthame, A. In, and P. Rascle, Some new Godunov and relaxation methods for two-phase flow problems, Godunov methods (Oxford, 1999), Kluwer/Plenum, New York, 2001, pp. 179-188. MR.1963591(2004a:76093)

[26] F. Coquel, E. Godlewski, and N. Seguin, Relaxation of fluid systems, Math. Models Methods Appl. Sci. 22 (2012), no. 8, 1250014, 52, DOI 10.1142/S0218202512500145. MR2928102

[27] F. Coquel and B. Perthame, Relaxation of energy and approximate Riemann solvers for general pressure laws in fluid dynamics, SIAM J. Numer. Anal. 35 (1998), no. 6, 2223-2249 (electronic), DOI 10.1137/S0036142997318528. MR1655844(2000a:76129)

[28] S. Diehl, A conservation law with point source and discontinuous flux function modelling continuous sedimentation, SIAM J. Appl. Math. 56 (1996), no. 2, 388-419, DOI 10.1137/S0036139994242425. MR1381652 (97a:35145)

[29] F. Dubois and P. LeFloch, Boundary conditions for nonlinear hyperbolic systems of conservation laws, J. Differential Equations 71 (1988), no. 1, 93-122, DOI 10.1016/00220396(88)90040-X. MR.922200(89c:35099)

[30] E. Duret, Y. Peysson, Q. H. Tran, P. Rouchon, Active bypass to eliminate severe-slugging in multiphase production, Proceedings of the 4th North American Conference on Multiphase Technology, Ban, June 2004, J. Brill, ed., Cranfield, 2004, BHR Group Limited, 205-223.

[31] M-P. Errera, S. Chemin, A fluid-solid thermal coupling applied to an effusion cooling system, 34th AIAA Computational Fluid Dynamics Conference, AIAA paper 2004-2140, 2004.

[32] M. Gad-el-Hak, Flow Control, Appl. Mech. Rev., 42, 261-293, 1989.

[33] M. Gad-el-Hak, D.M. Bushnell, Separation Control: Review, J. Fluids Eng., 113, 5-30, 1991.

[34] T. Galié, Couplage interfacial de modèles en dynamique des fluides. Application aux écoulements diphasiques, PhD thesis, Université Pierre et Marie Curie-Paris VI, France, 2009.

[35] S. Gavrilyuk and R. Saurel, Mathematical and numerical modeling of two-phase compressible flows with micro-inertia, J. Comput. Phys. 175 (2002), no. 1, 326-360, DOI 10.1006/jcph.2001.6951. MR.1877822(2002i:76110)

[36] J. Glimm, The interaction of nonlinear hyperbolic waves, Comm. Pure Appl. Math. 41 (1988), no. 5, 569-590, DOI 10.1002/cpa.3160410505. MR948072(89h:35198b)

[37] J. Glimm and D. H. Sharp, An S matrix theory for classical nonlinear physics, Found. Phys. 16 (1986), no. 2, 125-141, DOI 10.1007/BF01889377. MR836850 (88c:35141) 
[38] P. Goatin and P. G. LeFloch, The Riemann problem for a class of resonant hyperbolic systems of balance laws, Ann. Inst. H. Poincaré Anal. Non Linéaire 21 (2004), no. 6, 881-902, DOI 10.1016/j.anihpc.2004.02.002. MR2097035 (2006i:35225)

[39] E. Godlewski, Coupling fluid models. Exploring some features of interfacial coupling, Finite volumes for complex applications V, ISTE, London, 2008, pp. 87-102. MR2441448 (2009m:76063)

[40] E. Godlewski, K.-C. Le Thanh, and P.-A. Raviart, The numerical interface coupling of nonlinear hyperbolic systems of conservation laws. II. The case of systems, M2AN Math. Model. Numer. Anal. 39 (2005), no. 4, 649-692, DOI 10.1051/m2an:2005029. MR2165674 (2006h:65133)

[41] E. Godlewski and P.-A. Raviart, Hyperbolic systems of conservation laws, Mathématiques \& Applications (Paris) [Mathematics and Applications], vol. 3/4, Ellipses, Paris, 1991. MR 1304494 (95i:65146)

[42] E. Godlewski and P.-A. Raviart, Numerical approximation of hyperbolic systems of conservation laws, Applied Mathematical Sciences, vol. 118, Springer-Verlag, New York, 1996. MR.1410987 (98d:65109)

[43] E. Godlewski and P.-A. Raviart, The numerical interface coupling of nonlinear hyperbolic systems of conservation laws. I. The scalar case, Numer. Math. 97 (2004), no. 1, 81-130, DOI 10.1007/s00211-002-0438-5. MR2045460 (2005e:65130)

[44] L. Gosse, A well-balanced scheme using non-conservative products designed for hyperbolic systems of conservation laws with source terms, Math. Models Methods Appl. Sci. 11 (2001), no. 2, 339-365, DOI 10.1142/S021820250100088X. MR.1820677 (2002g:65097)

[45] L. Gosse, Localization effects and measure source terms in numerical schemes for balance laws, Math. Comp. 71 (2002), no. 238, 553-582, DOI 10.1090/S0025-5718-01-01354-0. MR.1885615 (2003e:65147)

[46] L. Gosse, Time-splitting schemes and measure source terms for a quasilinear relaxing system, Math. Models Methods Appl. Sci. 13 (2003), no. 8, 1081-1101, DOI 10.1142/S0218202503002829. MR1998816 (2005b:35175)

[47] L. Gosse, Computing Qualitatively Correct Approximations of Balance Laws, SIMAI Springer Series, vol. 2, Springer, Milan, 2013. Exponential-fit, well-balanced and asymptoticpreserving. MR3053000

[48] J. M. Greenberg and A. Y. Leroux, A well-balanced scheme for the numerical processing of source terms in hyperbolic equations, SIAM J. Numer. Anal. 33 (1996), no. 1, 1-16, DOI 10.1137/0733001. MR1377240 (97c:65144)

[49] G. Guerra, Well-posedness for a scalar conservation law with singular nonconservative source, J. Differential Equations 206 (2004), no. 2, 438-469, DOI 10.1016/j.jde.2004.04.008. MR2095821 (2005i:35173)

[50] J.-M. Hérard, A rough scheme to couple free and porous media, Int. J. Finite Vol. 3 (2006), no. 2, 28. MR2465466 (2009m:76098)

[51] J-M. Hérard, O. Hurisse, Coupling two and one-dimensional unsteady Euler equations through a thin interface, Computers \& Fluids, 36, 651-666, 2007.

[52] J.-M. Hérard and O. Hurisse, The numerical coupling of a two-fluid model with an homogeneous relaxation model, Finite volumes for complex applications V, ISTE, London, 2008, pp. 503-510. MR2451446

[53] S. Jin and Z. P. Xin, The relaxation schemes for systems of conservation laws in arbitrary space dimensions, Comm. Pure Appl. Math. 48 (1995), no. 3, 235-276, DOI 10.1002/cpa.3160480303. MR 1322811 (96c:65134)

[54] Y.-S. Kwon and A. Vasseur, Strong traces for solutions to scalar conservation laws with general flux, Arch. Ration. Mech. Anal. 185 (2007), no. 3, 495-513, DOI 10.1007/s00205007-0055-7. MR2322819(2008h:35234)

[55] T.-P. Liu, Hyperbolic conservation laws with relaxation, Comm. Math. Phys. 108 (1987), no. 1, 153-175. MR872145 (88f:35092)

[56] H. Mathis, C. Cancès, E. Godlewski, and N. Seguin, Dynamic model adaptation for multiscale simulation of hyperbolic systems with relaxation, J. Sci. Comput. 63 (2015), no. 3, 820-861, DOI 10.1007/s10915-014-9915-0. MR.3342726

[57] A.M. Mitchell, J. Delery, Research into Vortex Breakdown Control, Progress in Aerospace Sciences, 37, 385-418, 2001. 
[58] A. Murrone, P. Villedieu, Numerical modeling of dispersed two-phase flows, Aerospace Lab, $2,1-13,2011$.

[59] K. Saleh, Analyse et simulation numérique par relaxation d'écoulements diphasiques compressibles. Contribution au traitement des phases évanescentes, $\mathrm{PhD}$ thesis, Université Pierre et Marie Curie-Paris 6, France, 2012.

[60] I. Suliciu, On the thermodynamics of rate-type fluids and phase transitions. I. Rate-type fluids, Internat. J. Engrg. Sci. 36 (1998), no. 9, 921-947, DOI 10.1016/S0020-7225(98)000056. MR.1636396 (99f:76009)

[61] A. Vasseur, Well-posedness of scalar conservation laws with singular sources, Methods Appl. Anal. 9 (2002), no. 2, 291-312. MR 1957491 (2003k:35161)

[62] S. Tiwari and A. Klar, An adaptive domain decomposition procedure for Boltzmann and Euler equations, J. Comput. Appl. Math. 90 (1998), no. 2, 223-237, DOI 10.1016/S03770427(98)00027-2. MR 1624342(99c:76088)

CNRS and CMAP, Ecole polytechnique, 91128 Palaiseau Cedex, France

E-mail address: frederic.coquel@cmap.polytechnique.fr

Sorbonne Universités, UPMC Univ Paris 06, UMR 7598, Laboratoire Jacques-Louis Lions, F-75005, PARIS, France - AND — CNRS, UMR 7598, LABoratoire JaCQues-Louis Lions, F-75005, Paris, France, inRIA-Paris, EPC Ange, F-75012, Paris, France

E-mail address: edwige.godlewski@upmc.fr

Onera the French Aerospace lab, 92320 Châtillon Cedex, France - and — UPMC Université Paris 06, UMR 7598, Laboratoire JaCques-Louis Lions, F-75005, Paris, France E-mail address: khalil.haddaoui.edu@gmail.com

Onera The French Aerospace Lab, 92320 Châtillon Cedex, France

E-mail address: claude.marmignon@onera.fr

Onera The French Aerospace Lab, 92320 Châtillon Cedex, France

E-mail address: florent.renac@onera.fr 